\title{
On the supersymmetric extension of asymptotic symmetries in three spacetime dimensions
}

\author{
Ricardo Caroca $^{1, \mathrm{a}}$, Patrick Concha ${ }^{1, \mathrm{~b}}$, Octavio Fierro $^{1, \mathrm{c}}$, Evelyn Rodríguez $^{2, \mathrm{~d}}$ \\ ${ }^{1}$ Departamento de Matemática y Física Aplicadas, Universidad Católica de la Santísima Concepción, Alonso de Ribera 2850, Concepción, Chile \\ ${ }^{2}$ Departamento de Ciencias, Facultad de Artes Liberales, Universidad Adolfo Ibáñez, Viña del Mar, Chile
}

Received: 28 August 2019 / Accepted: 26 December 2019 / Published online: 11 January 2020

(C) The Author(s) 2020

\begin{abstract}
In this work we obtain known and new supersymmetric extensions of diverse asymptotic symmetries defined in three spacetime dimensions by considering the semigroup expansion method. The super- $B M S_{3}$, the superconformal algebra and new infinite-dimensional superalgebras are obtained by expanding the super-Virasoro algebra. The new superalgebras obtained are supersymmetric extensions of the asymptotic algebras of the Maxwell and the $\mathfrak{s o}(2,2) \oplus$ $\mathfrak{s o}(2,1)$ gravity theories. We extend our results to the $\mathcal{N}=2$ and $\mathcal{N}=4$ cases and find that R-symmetry generators are required. We also show that the new infinite-dimensional structures are related through a flat limit $\ell \rightarrow \infty$.
\end{abstract}

\section{Contents}

1 Introduction ................

2 The semigroup expansion method and Super-Virasoro algebra ................. 2

3 Known examples . . . . . . . . . . . . . . 3

3.1 Super- $B M S_{3}$ algebra ................... 3

3.2 Superconformal algebra . . . . . . . . . . . . . 4

$3.3(1,1)$ superconformal algebra . . . . . . . . . 6

4 New examples ................. 6

4.1 Supersymmetric extension of the asymptotic algebra of the Maxwell gravity theory . . . . 6 4.1.1 Minimal deformed super- $\widetilde{B M} S_{3}$ algebra . 7 4.1.2 $\mathcal{N}=2$ deformed super- $-\widetilde{M M} S_{3}$ algebra . . 8 4.1.3 $\mathcal{N}=4$ deformed super- $-\widetilde{M} S_{3}$ algebra . . 9

4.2 Supersymmetric extension of the asymptotic algebra of the $\mathfrak{s o}(2,2) \oplus \mathfrak{s o}(2,1)$ gravity theory 10 4.2.1 Minimal enlarged super- $B M S_{3}$ algebra . 11

\footnotetext{
a e-mail: rcaroca@ucsc.cl

b e-mail: patrick.concha@ucsc.cl

c e-mail: ofierro@ucsc.cl

de-mail: evelyn.rodriguez@edu.uai.cl
}

4.2.2 $\mathcal{N}=2$ enlarged super- $B M S_{3}$ algebra $\ldots 12$

4.2.3 $\mathcal{N}=4$ enlarged super- $B M S_{3}$ algebra $\ldots 14$

4.2.4 Non-standard enlarged super- $B M S_{3}$ alge-

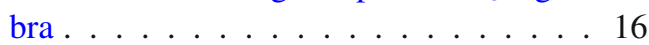

5 Conclusions ............... . . 18

A Appendix . . . . . . . . . . . . . . . . . . . . 19

B Appendix . . . . . . . . . . . . . . . . . . 19

References . . . . . . . . . . . . . . . 20

\section{Introduction}

In recent years, infinite-dimensional symmetries have received a growing interest in the study of fluid mechanics, string theory, two-dimensional field theory, soliton theory and gravity theory among others. In particular, the infinite-dimensional (super)symmetries of the Virasoro type result to describe the boundary dynamics of three-dimensional (super)gravity theories. In particular, three-dimensional theories are worth to study and are interesting toy models since they could be useful to approach and understand open issues in higherdimensional cases.

At the bosonic level, the asymptotic symmetry of a threedimensional gravity in presence of a negative cosmological constant corresponds to two copies of the Virasoro algebra [1]. Such structure is obtained by considering suitable boundary conditions. In the vanishing-cosmological constant case, the symmetry of asymptotically flat spacetimes at null infinity is described by the $B M S_{3}$ algebra [2-4] which is the three-dimensional version of the $B M S$ algebra introduced more than a half century ago $[5,6]$. Extensions of the $B M S_{3}$ symmetry have been subsequently studied in [7-16].

More recently, an extended and deformed $B M S_{3}$ algebra (which we have called deformed $\widehat{B M S}_{3}$ algebra) appears as the asymptotic symmetry of a three-dimensional gravity theory invariant under the so-called Maxwell algebra [17]. The Maxwell symmetry has been presented in [18-20] in order to describe the presence of a constant electromagnetic field 
background in Minkowski space. Such symmetry has then been generalized by diverse authors with different applications [21-33]. Subsequently, a semi-simple enlargement of the $B M S_{3}$ algebra has been introduced in [34] corresponding to the asymptotic symmetry of a gravity theory invariant under the so-called AdS-Lorentz algebra which can be seen as a semi-simple enlargement of the Poincaré algebra. The AdS-Lorentz symmetry has been introduced in [35-38] and has lead to diverse applications in the context of Lovelock gravity [39-41] and non-relativistic gravity theory [42]. An interesting feature of the enlarged $B M S_{3}$ algebra obtained in [34] is the connection to the deformed $\overparen{B M S_{3}}$ algebra through the flat limit $\ell \rightarrow \infty$.

A minimal supersymmetric extension of the $B M S_{3}$ algebra has been shown to describe the asymptotic structure of the $\mathcal{N}=1$ supergravity in three spacetime dimensions considering suitable boundary conditions [43]. The extensions to $\mathcal{N}=2$ [44-46], $\mathcal{N}=4$ [47] and $\mathcal{N}=8$ [48] have later been explored by diverse authors. On the other hand, the supersymmetric extensions of the so-called deformed $\widehat{B M S_{3}}$ algebra and the semi-simple enlargement of the $B M S_{3}$ algebra remain unknown. Interestingly, the $B M S_{3}$, the deformed $\widetilde{B M S}_{3}$ and the enlarged $B M S_{3}$ algebras can alternatively be recovered by applying a semigroup expansion [49] ( $S$ expansion) to the Virasoro algebra [50]. Furthermore, the super- $B M S_{3}$ algebra and its $\mathcal{N}$-extended versions have been recently obtained applying the $S$-expansion to the superVirasoro algebra [51].

In this paper, we extend the approach of $[50,51]$ to others asymptotic symmetries whose supersymmetric extensions are unknown. In particular, we apply the $S$-expansion to the super-Virasoro algebra in order to introduce novel supersymmetric extensions of known asymptotic symmetries. The new infinite-dimensional superalgebras obtained correspond to the supersymmetric extensions of the deformed $\widehat{B M S_{3}}$ algebra and the enlarged $B M S_{3}$ algebra. Interestingly, they can be seen as the infinite-dimensional lifts of the Maxwell and AdS-Lorentz superalgebra introduced in [52] and [53], respectively. Furthermore, as their respective finite subalgebras, they are related through a flat limit $\ell \rightarrow \infty$. We extend our results to the $\mathcal{N}=2$ and $\mathcal{N}=4$ cases and show that the new $\mathcal{N}$-extended infinite-dimensional superalgebras require the presence of R-symmetry generators.

The paper is organized as follows: In Sect. 2 we give a brief review of the $S$-expansion procedure and the superVirasoro algebra. Sections 3 and 4 contain our main results. In Sect. 3, we show that known asymptotic supersymmetries can alternatively be recovered using the $S$-expansion method. In Sect. 4, we present novel supersymmetric extensions of the deformed and enlarged $B M S_{3}$ algebras applying different semigroups to the super-Virasoro algebra. The extensions to $\mathcal{N}=2$ and $\mathcal{N}=4$ are also considered. Section 5 is devoted to discussion and possible developments.

\section{The semigroup expansion method and Super-Virasoro algebra}

The Lie algebra expansion method was first introduced in [54] and subsequently developed in [55-57] in the context of three-dimensional Chern-Simons (CS) supergravity and M-theory superalgebra. A generalization of the expansion procedure using semigroups was later presented in [49]. Such Abelian semigroup expansion method consists in combining the elements of a semigroup $S$ with the structure constants of a Lie algebra $\mathfrak{g}$ in order to obtain a new expanded Lie algebra $\mathfrak{G}=S \times \mathfrak{g}$. The $S$-expanded Lie algebra satisfies

$\left[T_{(A, \alpha)}, T_{(B, \beta)}\right]=K_{\alpha \beta}^{\gamma} C_{A B}^{C} T_{(C, \gamma)}$,

where $T_{(A, \alpha)}=\lambda_{\alpha} T_{A}$ are the generators of the expanded algebra $\mathfrak{G}$ defined in terms of the generators $T_{A}$ of the original algebra $\mathfrak{g}$ and in terms of the elements $\lambda_{\alpha}$ of the semigroup $S$. Here $C_{A B}^{C}$ are the structure constants of the original algebra $\mathfrak{g}$ while $K_{\alpha \beta}^{\gamma}$ is the so-called 2-selector defined by

$K_{\alpha \beta}^{\gamma}=\left\{\begin{array}{l}1 \quad \text { when } \lambda_{\alpha} \lambda_{\beta}=\lambda_{\gamma} \\ 0 \text { otherwise. }\end{array}\right.$

Then, the $S$-expanded algebra $\mathfrak{G}=S \times \mathfrak{g}$ is a Lie algebra with structure constants

$C_{(A, \alpha)(B, \beta)}^{(C, \gamma)}=K_{\alpha \beta}^{\gamma} C_{A B}^{C}$.

Interestingly, it is possible to extract a smaller algebra of the expanded one when the semigroup has a zero element $0_{S} \in S$. In particular, the algebra obtained by imposing the condition $0_{S} T_{A}=0$ on the expanded algebra $\mathfrak{G}$ is called $0_{S}$-reduced algebra of $\mathfrak{G}$.

There is an alternative procedure to extract smaller algebra which requires to consider a decomposition of the original algebra $\mathfrak{g}$ and of the semigroup $S$. Let $\mathfrak{g}=\bigoplus_{p \in I} V_{p}$ be a subspace decomposition of the original algebra $\mathfrak{g}$, where $I$ denotes a set of indices. Then for each $p, q \in I$ it is possible to define $i_{(p, q)} \subset I$ such that

$\left[V_{p}, V_{q}\right] \subset \bigoplus_{r \in i_{(p, q)}} V_{r}$.

On the other hand, let us consider a subset decomposition of the semigroup $S=\bigcup_{p \in I} S_{p}$ such that

$S_{p} \cdot S_{q} \subset \bigcap_{r \in i_{(p, q)}} S_{r}$

When such subset decomposition exists, we say that it is in resonance with the subspace decomposition of the algebra $\mathfrak{g}$. In particular, the subalgebra 
$\mathfrak{G}_{R}=\bigoplus_{p \in I} S_{p} \times V_{p}$

is called the resonant subalgebra of $\mathfrak{G}$. Further studies of the $S$-expansion method have been developed by diverse authors and can be found in [58-66].

It is interesting to notice that non-trivial (anti-)commutation relations can be obtained by choosing suitable semigroups $S$ and a pertinent Lie (super)algebra as the original (super)algebra $\mathfrak{g}$. Here we shall consider a particular infinitedimensional superalgebra as our starting point of our construction. Let $\mathfrak{g}$ be the super-Virasoro algebra, which we shall denote as $\mathfrak{s v i r}$ and whose generators satisfy the following (anti-)commutation relations:

$\left[\ell_{m}, \ell_{n}\right]=(m-n) \ell_{m+n}+\frac{c}{12} m\left(m^{2}-1\right) \delta_{m+n, 0}$,

$\left[\ell_{m}, \mathcal{Q}_{r}\right]=\left(\frac{m}{2}-r\right) \mathcal{Q}_{m+r}$,

$\left\{\mathcal{Q}_{r}, \mathcal{Q}_{s}\right\}=\ell_{r+s}+\frac{c}{6}\left(r^{2}-\frac{1}{4}\right) \delta_{r+s, 0}$,

where $c$ is a central extension. Such infinite-dimensional algebra can be decomposed in subspaces as

$\mathfrak{s v i r}=V_{0} \oplus V_{1}$,

where $V_{0}$ is spanned by the bosonic generators, while $V_{1}$ is the fermionic subspace. One can note that the subspaces satisfy a graded Lie algebra,

$$
\begin{aligned}
& {\left[V_{0}, V_{0}\right] \subset V_{0},} \\
& {\left[V_{0}, V_{1}\right] \subset V_{1},} \\
& {\left[V_{1}, V_{1}\right] \subset V_{0} .}
\end{aligned}
$$

Let us note that there is a finite subalgebra spanned by the generators $\ell_{0}, \ell_{1}, \ell_{-1}, Q_{ \pm \frac{1}{2}}$ which are related to the superLorentz generators through the following change of basis:

$$
\begin{aligned}
& \ell_{-1}=-\sqrt{2} M_{0}, \ell_{1}=\sqrt{2} M_{1}, \quad \ell_{0}=M_{2}, \\
& \mathcal{Q}_{-\frac{1}{2}}=\sqrt{2} \tilde{Q}_{+}, \quad \mathcal{Q}_{\frac{1}{2}}=\sqrt{2} \tilde{Q}_{-} .
\end{aligned}
$$

The supersymmetric extension of the Lorentz algebra has been introduced in [67] and can be used to construct an exotic supersymmetric CS action [51].

An $S$-expanded super-Virasoro algebra can be obtained by considering a semigroup $S=\left\{\lambda_{i}\right\}$ such that the new algebra is given by the direct product $S \times \mathfrak{s v i r}$. The expanded generators are given in terms of the super-Virasoro ones as

$\ell_{(m, \alpha)}=\lambda_{\alpha} \ell_{m}$,

$Q_{(r, \alpha)}=\lambda_{\alpha} Q_{r}$,

and satisfy the (anti-)commutation relations

$$
\begin{aligned}
{\left[\ell_{(m, \alpha)}, \ell_{(n, \beta)}\right]=} & (m-n) K_{\alpha \beta}^{\gamma} \ell_{(m+n, \gamma)} \\
& +\frac{c_{\alpha \beta}}{12} m\left(m^{2}-1\right) \delta_{m+n, 0},
\end{aligned}
$$

$\left[\ell_{(m, \alpha)}, \mathcal{Q}_{(r, \beta)}\right]=\left(\frac{m}{2}-r\right) K_{\alpha \beta}^{\gamma} \mathcal{Q}_{(m+r, \gamma)}$,

$\left\{\mathcal{Q}_{(r, \alpha)}, \mathcal{Q}_{(s, \beta)}\right\}=K_{\alpha \beta}^{\gamma} \ell_{(r+s, \gamma)}+\frac{c_{\alpha \beta}}{6}\left(r^{2}-\frac{1}{4}\right) \delta_{r+s, 0}$.

Here, $c_{\alpha \beta}$ denotes a set of central charges given by

$c_{\alpha \beta}=c K_{\alpha \beta}^{\gamma} \lambda_{\gamma}$.

Interestingly, the $S$-expanded super-Virasoro algebra has a finite subalgebra which results to be the direct product $S \times \mathcal{S L}$ where $\mathcal{S} \mathcal{L}$ is the finite super-Lorentz subalgebra of the original super-Virasoro algebra. Thus, the semigroup $S$ which allows us to obtain a new finite (super)algebra also reproduces its infinite-dimensional version. Such particularity has first been observed at the bosonic level in [50] and subsequently noticed at the supersymmetric level in [51].

In what follows, we shall first recover known asymptotic supersymmetries using the semigroup expansion method. We then extend our methodology to obtain new supersymmetric extension of particular asymptotic symmetries. Naturally, in order to get $\mathcal{N}$-extended superalgebras we shall require to consider an $\mathcal{N}$-extended super-Virasoro algebra as the original superalgebra.

\section{Known examples}

It is well known that in asymptotically flat spacetimes, the $B M S_{3}$ algebra describes the asymptotic symmetry of General Relativity in three spacetime dimensions [2-4]. At the supersymmetric level, the super- $B M S_{3}$ algebra appears as the asymptotic symmetry of a three-dimensional minimal supergravity for a suitable set of asymptotically flat boundary conditions [43]. The $\mathcal{N}$-extension of the $B M S_{3}$ algebra has subsequently been explored by diverse authors in [4448]. In particular, the $\mathcal{N}$-extended super- $B M S_{3}$ algebras can alternatively be obtained by performing a suitable contraction of the $\mathcal{N}$-extended superconformal algebras [68].

Recently, it has been shown in [51] that the $\mathcal{N}$-extended super- $B M S_{3}$ algebra can also be recovered through the semigroup expansion method considering an $\mathcal{N}$-extended superVirasoro algebra as the original superalgebra. Here we briefly review the obtention of the super- $B M S_{3}$ algebra following [51]. Then, we show that the superconformal algebra can also be obtained considering a different semigroup.

\subsection{Super- $B M S_{3}$ algebra}

As was shown in [51], the super- $B M S_{3}$ algebra appears as an $S$-expansion of the super-Virasoro algebra (2.7). This can be done by considering $S_{E}^{(2)}=\left\{\lambda_{0}, \lambda_{1}, \lambda_{2}, \lambda_{3}\right\}$ as the relevant Abelian semigroup whose elements satisfy 
$\lambda_{3} \mid \begin{array}{llll}\lambda_{3} & \lambda_{3} & \lambda_{3} & \lambda_{3}\end{array}$

$\lambda_{2} \lambda_{2} \lambda_{3} \lambda_{3} \lambda_{3}$

$\lambda_{1} \lambda_{1} \lambda_{2} \lambda_{3} \lambda_{3}$

\begin{tabular}{l|llll}
$\lambda_{0}$ & $\lambda_{0}$ & $\lambda_{1}$ & $\lambda_{2}$ & $\lambda_{3}$ \\
\hline & $\lambda_{0}$ & $\lambda_{1}$ & $\lambda_{2}$ & $\lambda_{3}$
\end{tabular}

where $\lambda_{3}=0_{S}$ is the zero element of the semigroup. A particular subset decomposition $S_{E}^{(2)}=S_{0} \cup S_{1}$, with

$S_{0}=\left\{\lambda_{0}, \lambda_{2}, \lambda_{3}\right\}$,

$S_{1}=\left\{\lambda_{1}, \lambda_{3}\right\}$,

is said to be resonant since it satisfies the same structure than the subspaces (2.9),

$S_{0} \cdot S_{0} \subset S_{0}$,

$S_{0} \cdot S_{1} \subset S_{1}$,

$S_{1} \cdot S_{1} \subset S_{0}$.

The minimal super- $B M S_{3}$ algebra is obtained after extracting a resonant subalgebra of $S_{E}^{(2)} \times \mathfrak{s v i r}$ and performing a $0_{S}$-reduction. In particular, the super- $B M S_{3}$ generators are related to the super-Virasoro ones through

$\mathcal{J}_{m}=\lambda_{0} \ell_{m}, c_{1}=\lambda_{0} c$

$\mathcal{P}_{m}=\lambda_{2} \ell_{m}, c_{2}=\lambda_{2} c$,

$\mathcal{G}_{r}=\lambda_{1} \mathcal{Q}_{r}$.

Then, using the multiplication law of the semigroup (3.1) and the (anti-)commutation relations of the super-Virasoro algebra (2.7), one finds the (anti-)commutators of the minimal super- $B M S_{3}$ algebra with two central charges [43,69]:

$$
\begin{aligned}
& {\left[\mathcal{J}_{m}, \mathcal{J}_{n}\right]=(m-n) \mathcal{J}_{m+n}+\frac{c_{1}}{12}\left(m^{3}-m\right) \delta_{m+n, 0},} \\
& {\left[\mathcal{J}_{m}, \mathcal{P}_{n}\right]=(m-n) \mathcal{P}_{m+n}+\frac{c_{2}}{12}\left(m^{3}-m\right) \delta_{m+n, 0},} \\
& {\left[\mathcal{J}_{m}, \mathcal{G}_{r}\right]=\left(\frac{m}{2}-r\right) \mathcal{G}_{m+r},} \\
& \left\{\mathcal{G}_{r}, \mathcal{G}_{s}\right\}=\mathcal{P}_{r+s}+\frac{c_{2}}{6}\left(r^{2}-\frac{1}{4}\right) \delta_{r+s, 0} .
\end{aligned}
$$

The central charges $c_{1}$ and $c_{2}$ are related to the $\mathcal{N}=1 \mathrm{CS}$ Poincaré supergravity action with

$c_{1}=12 k \alpha_{0}, \quad c_{2}=12 k \alpha_{1}$,

where $\alpha_{0}$ and $\alpha_{1}$ are the respective coupling constants of the exotic CS term and the Einstein-Hilbert term, respectively. It is interesting to note that the super- $B M S_{3}$ algebra (3.6) is isomorphic to the supersymmetric extension of the twodimensional Galilean conformal algebra introduced in [70, 71].

One can notice that the super- $B M S_{3}$ algebra has a finite subalgebra spanned by $\mathcal{J}_{0}, \mathcal{J}_{1}, \mathcal{J}_{-1}, \mathcal{P}_{0}, \mathcal{P}_{1}, \mathcal{P}_{-1}$ and $\mathcal{G}_{\frac{1}{2}}$, $\mathcal{G}_{-\frac{1}{2}}$ which are related to the Poincaré superalgebra through the following change of basis:

$$
\begin{aligned}
& \mathcal{J}_{-1}=-\sqrt{2} J_{0}, \quad \mathcal{J}_{1}=\sqrt{2} J_{1}, \quad \mathcal{J}_{0}=J_{2}, \\
& \mathcal{P}_{-1}=-\sqrt{2} P_{0}, \quad \mathcal{P}_{1}=\sqrt{2} P_{1}, \quad \mathcal{P}_{0}=P_{2}, \\
& \mathcal{G}_{-\frac{1}{2}}=\sqrt{2} Q_{+}, \quad \mathcal{G}_{\frac{1}{2}}=\sqrt{2} Q_{-},
\end{aligned}
$$

and using a non-diagonal Minkowski metric

$\eta_{a b}=\left(\begin{array}{lll}0 & 1 & 0 \\ 1 & 0 & 0 \\ 0 & 0 & 1\end{array}\right)$

Interestingly, such subalgebra can also be obtained by considering the $S_{E}^{(2)}$-expansion of the super-Lorentz subalgebra of the super-Virasoro. Indeed, as was shown in [51], the super Poincaré structure appears as an $S_{E}^{(2)}$-expansion of the superLorentz algebra. Such particularity was also observed at the bosonic level for the $B M S_{3}$ algebra in [50]. An alternative procedure to derive the $B M S_{3}$ algebra using an algebraic operation can be found in [72].

The generalizations to $\mathcal{N}=2$ and $\mathcal{N}=4$ using the semigroup expansion method can be found in [51].

\subsection{Superconformal algebra}

The superconformal algebra with $(1,0)$ supersymmetry can be obtained by considering a particular $S$-expansion of the super-Virasoro algebra (2.7). In fact, let $S_{L}^{(1)}=\left\{\lambda_{0}, \lambda_{1}, \lambda_{2}\right\}$ be the relevant semigroup whose elements satisfy the following multiplication law

\begin{tabular}{l|lll}
$\lambda_{2}$ & $\lambda_{2}$ & $\lambda_{2}$ & $\lambda_{2}$ \\
$\lambda_{1}$ & $\lambda_{2}$ & $\lambda_{1}$ & $\lambda_{2}$ \\
$\lambda_{0}$ & $\lambda_{0}$ & $\lambda_{2}$ & $\lambda_{2}$ \\
\hline & $\lambda_{0}$ & $\lambda_{1}$ & $\lambda_{2}$
\end{tabular}

with $\lambda_{2}=0_{S}$ being the zero element of the semigroup. Let us consider now a resonant decomposition of the semigroup

$S_{0}=\left\{\lambda_{0}, \lambda_{1}, \lambda_{2}\right\}$,

$S_{1}=\left\{\lambda_{1}, \lambda_{2}\right\}$,

which satisfies

$S_{0} \cdot S_{0} \subset S_{0}$,

$S_{0} \cdot S_{1} \subset S_{1}$,

$S_{1} \cdot S_{1} \subset S_{0}$.

Then, the resonant subalgebra is given by

$W_{R}=W_{0} \oplus W_{1}=S_{0} \times V_{0} \oplus S_{1} \times V_{1}$,

with $V_{0}$ and $V_{1}$ being the subspaces of the super-Virasoro algebra. The superconformal algebra is obtained after performing a $0_{S}$-reduction whose generators are related to the super-Virasoro ones through 
$\overline{\mathcal{L}}_{m}=\lambda_{0} \ell_{m}, \bar{c}=\lambda_{0} c$,

$\mathcal{L}_{m}=\lambda_{1} \ell_{m}, c=\lambda_{1} c$,

$\mathcal{Q}_{r}=\lambda_{1} \mathcal{Q}_{r}$.

In particular, using the multiplication law of the semigroup (3.9) and the (anti-)commutation relations of the superVirasoro algebra (2.7), one finds the (anti-)commutators of the superconformal algebra:

$$
\begin{aligned}
& {\left[\mathcal{L}_{m}, \mathcal{L}_{n}\right]=(m-n) \mathcal{L}_{m+n}+\frac{c}{12} m\left(m^{2}-1\right) \delta_{m+n, 0}} \\
& {\left[\overline{\mathcal{L}}_{m}, \overline{\mathcal{L}}_{n}\right]=(m-n) \overline{\mathcal{L}}_{m+n}+\frac{\bar{c}}{12} m\left(m^{2}-1\right) \delta_{m+n, 0}} \\
& {\left[\mathcal{L}_{m}, \mathcal{Q}_{r}\right]=\left(\frac{m}{2}-r\right) \mathcal{Q}_{m+r}} \\
& \left\{\mathcal{Q}_{r}, \mathcal{Q}_{s}\right\}=\mathcal{L}_{r+s}+\frac{c}{6}\left(r^{2}-\frac{1}{4}\right) \delta_{r+s, 0}
\end{aligned}
$$

Such infinite-dimensional superalgebra corresponds to two copies of the Virasoro algebra, one of which is supersymmetric, and results to be the corresponding asymptotic symmetry of the three-dimensional supergravity theory.

Let us note that a flat limit can be performed by considering first a redefinition of the generators,

$$
\begin{aligned}
\mathcal{J}_{m} & =\mathcal{L}_{m}-\overline{\mathcal{L}}_{-m}, \quad \mathcal{P}_{m}=\frac{1}{\ell}\left(\mathcal{L}_{m}+\overline{\mathcal{L}}_{-m}\right), \\
\mathcal{G}_{r} & =\sqrt{\frac{2}{\ell}} \mathcal{Q}_{r},
\end{aligned}
$$

which allows us to rewrite the superconformal algebra as the infinite-dimensional lift of the AdS superalgebra

$$
\begin{aligned}
& {\left[\mathcal{J}_{m}, \mathcal{J}_{n}\right]=(m-n) \mathcal{J}_{m+n}+\frac{c_{1}}{12} m\left(m^{2}-1\right) \delta_{m+n, 0},} \\
& {\left[\mathcal{J}_{m}, \mathcal{P}_{n}\right]=(m-n) \mathcal{P}_{m+n}+\frac{c_{2}}{12} m\left(m^{2}-1\right) \delta_{m+n, 0},} \\
& {\left[\mathcal{P}_{m}, \mathcal{P}_{n}\right]=\frac{1}{\ell^{2}}(m-n) \mathcal{J}_{m+n}}
\end{aligned}
$$

$$
\begin{aligned}
\left\{\mathcal{G}_{r}, \mathcal{G}_{s}\right\}= & \frac{\mathcal{J}_{r+s}}{\ell}+\mathcal{P}_{r+s} \\
& +\frac{\left(c_{1} / \ell+c_{2}\right)}{6}\left(r^{2}-\frac{1}{4}\right) \delta_{r+s, 0} .
\end{aligned}
$$

where we have defined $c_{1}=c-\bar{c}$ and $c_{2}=\frac{1}{\ell}(c+\bar{c})$. In particular, the central charges $c_{1}$ and $c_{2}$ are related to the minimal CS AdS supergravity action with

$c_{1}=12 k \alpha_{0}, \quad c_{2}=12 k \alpha_{1}$,

where $\alpha_{0}$ and $\alpha_{1}$ are the respective coupling constant of the exotic Lagrangian term and Einstein-Hilbert term. One can see that the superconformal algebra in the basis $\left\{\mathcal{J}_{m}, \mathcal{P}_{m}, \mathcal{G}_{r}\right\}$ reproduces the super- $B M S_{3}$ algebra (3.6) in the flat limit $\ell \rightarrow \infty$.

Let us note that the superconformal algebra (3.16) contains a finite subalgebra spanned by $\mathcal{J}_{0}, \mathcal{J}_{1}, \mathcal{J}_{-1}, \mathcal{P}_{0}, \mathcal{P}_{1}, \mathcal{P}_{-1}$ and $\mathcal{G}_{\frac{1}{2}}, \mathcal{G}_{-\frac{1}{2}}$ which are related to the AdS superalgebra through the following change of basis:

$$
\begin{aligned}
& \mathcal{J}_{-1}=-\sqrt{2} J_{0}, \quad \mathcal{J}_{1}=\sqrt{2} J_{1}, \quad \mathcal{J}_{0}=J_{2}, \\
& \mathcal{P}_{-1}=-\sqrt{2} P_{0}, \mathcal{P}_{1}=\sqrt{2} P_{1}, \quad \mathcal{P}_{0}=P_{2}, \\
& \mathcal{G}_{-\frac{1}{2}}=\sqrt{2} Q_{+}, \quad \mathcal{G}_{\frac{1}{2}}=\sqrt{2} Q_{-} \text {. }
\end{aligned}
$$

As it is well known, the generators of the AdS superalgebra can be rewritten as two copies of the Lorentz algebra, one of which is augmented by supersymmetry. Such superalgebra, which results to be the finite subalgebra of the superconformal algebra in the basis $\left\{\mathcal{L}_{m}, \overline{\mathcal{L}}_{m}, \mathcal{Q}_{r}\right\}$, appears as a $0_{S}$-reduced resonant expansion of the super Lorentz algebra using the same semigroup $S_{L}^{(1)}$.

The following diagram summarizes our construction and the relationship between algebras:

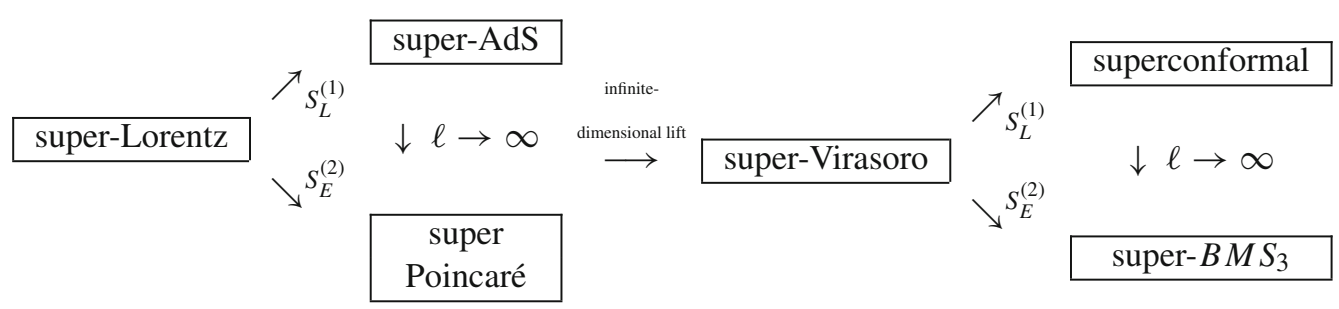

Along the paper we shall see that such diagram can be generalized to an enlarged symmetry. In particular, the election of the semigroups is based on those used to relate finite superalgebras. As we shall see, new infinite-dimensional algebras can be obtained using the same semigroups used at the finite level. 


\section{$3.3(1,1)$ superconformal algebra}

For completeness we extend our method to obtain the $(1,1)$ superconformal algebra. This can be done by considering the same semigroup $S_{L}^{(1)}=\left\{\lambda_{0}, \lambda_{1}, \lambda_{2}\right\}$ but without considering a resonant decomposition as in the previous case. Indeed, by considering the $S_{L}^{(1)}$-expansion of the super-Virasoro algebra we have

$S_{L}^{(1)} \times \mathfrak{s v i r}=\left\{\lambda_{0} \ell_{m}, \lambda_{1} \ell_{m}, \lambda_{2} \ell_{m}, \lambda_{0} \mathcal{Q}_{r}, \lambda_{1} \mathcal{Q}_{r}, \lambda_{2} \mathcal{Q}_{r}\right\}$,

Then, the $(1,1)$ superconformal algebra is obtained by performing the $0_{S}$-reduction with $\lambda_{2}=0_{S}$. In particular, the $(1,1)$ superconformal generators are related to the superVirasoro ones through

$\overline{\mathcal{L}}_{m}=\lambda_{0} \ell_{m}, \bar{c}=\lambda_{0} c$,

$\mathcal{L}_{m}=\lambda_{1} \ell_{m}, c=\lambda_{1} c$,

$\mathcal{Q}_{r}=\lambda_{1} \mathcal{Q}_{r}, \overline{\mathcal{Q}}_{r}=\lambda_{0} \mathcal{Q}_{r}$

The (anti-)commutators of the expanded superalgebra appears using the multiplication law of the semigroup (3.9) and the original commutators of the super-Virasoro algebra:

$$
\begin{aligned}
{\left[\mathcal{L}_{m}, \mathcal{L}_{n}\right] } & =(m-n) \mathcal{L}_{m+n}+\frac{c}{12} m\left(m^{2}-1\right) \delta_{m+n, 0}, \\
{\left[\overline{\mathcal{L}}_{m}, \overline{\mathcal{L}}_{n}\right] } & =(m-n) \overline{\mathcal{L}}_{m+n}+\frac{\bar{c}}{12} m\left(m^{2}-1\right) \delta_{m+n, 0}, \\
{\left[\mathcal{L}_{m}, \mathcal{Q}_{r}\right] } & =\left(\frac{m}{2}-r\right) \mathcal{Q}_{m+r},\left[\overline{\mathcal{L}}_{m}, \overline{\mathcal{Q}}_{r}\right] \\
& =\left(\frac{m}{2}-r\right) \overline{\mathcal{Q}}_{m+r}, \\
\left\{\mathcal{Q}_{r}, \mathcal{Q}_{s}\right\} & =\mathcal{L}_{r+s}+\frac{c}{6}\left(r^{2}-\frac{1}{4}\right) \delta_{r+s, 0}, \\
\left\{\overline{\mathcal{Q}}_{r}, \overline{\mathcal{Q}}_{s}\right\} & =\overline{\mathcal{L}}_{r+s}+\frac{\bar{c}}{6}\left(r^{2}-\frac{1}{4}\right) \delta_{r+s, 0},
\end{aligned}
$$

which corresponds to two copies of the super-Virasoro algebra. Let us note that such structure can also be derived from the $\mathcal{N}=(2,0)$ superconformal algebra [68].

Interestingly, the $\mathcal{N}=2$ super- $B M S_{3}$ algebra can be recovered as a flat limit after a suitable redefinition of the generators. In fact, the $(1,1)$ superconformal algebra can be rewritten as the infinite-dimensional lift of the $\mathcal{N}=2$ superAdS algebra

$$
\begin{aligned}
& {\left[\mathcal{J}_{m}, \mathcal{J}_{n}\right]=(m-n) \mathcal{J}_{m+n}+\frac{c_{1}}{12} m\left(m^{2}-1\right) \delta_{m+n, 0},} \\
& {\left[\mathcal{J}_{m}, \mathcal{P}_{n}\right]=(m-n) \mathcal{P}_{m+n}+\frac{c_{2}}{12} m\left(m^{2}-1\right) \delta_{m+n, 0},} \\
& {\left[\mathcal{P}_{m}, \mathcal{P}_{n}\right]=\frac{1}{\ell^{2}}(m-n) \mathcal{J}_{m+n}+\frac{c_{1}}{12 \ell^{2}} m\left(m^{2}-1\right) \delta_{m+n, 0},} \\
& {\left[\mathcal{J}_{m}, \mathcal{G}_{r}^{i}\right]=\left(\frac{m}{2}-r\right) \mathcal{G}_{m+r}^{i},}
\end{aligned}
$$

$$
\begin{aligned}
{\left[\mathcal{P}_{m}, \mathcal{G}_{r}^{i}\right]=} & \frac{1}{\ell}\left(\frac{m}{2}-r\right) \mathcal{G}_{m+r}^{i}, \\
\left\{\mathcal{G}_{r}^{i}, \mathcal{G}_{s}^{j}\right\}= & \delta^{i j}\left[\frac{\mathcal{J}_{r+s}}{\ell}+\mathcal{P}_{r+s}\right. \\
& \left.+\frac{\left(c_{1} / \ell+c_{2}\right)}{6}\left(r^{2}-\frac{1}{4}\right) \delta_{r+s, 0}\right] .
\end{aligned}
$$

where we have considered the following redefinitions of the generators,

$$
\begin{aligned}
& \mathcal{J}_{m}=\mathcal{L}_{m}-\overline{\mathcal{L}}_{-m}, \quad \mathcal{P}_{m}=\frac{1}{\ell}\left(\mathcal{L}_{m}+\overline{\mathcal{L}}_{-m}\right), \\
& \mathcal{G}_{r}^{1}=\sqrt{\frac{2}{\ell}} \mathcal{Q}_{r}, \quad \mathcal{G}_{r}^{2}=\sqrt{\frac{2}{\ell}} \overline{\mathcal{Q}}_{-r},
\end{aligned}
$$

with $c_{1}=c-\bar{c}$ and $c_{2}=\frac{1}{\ell}(c+\bar{c})$. Let us note that the $(1,1)$ superconformal algebra written in the basis $\left\{\mathcal{J}_{m}, \mathcal{P}_{m}, \mathcal{G}_{r}^{i}\right\}$ leads to the $\mathcal{N}=2$ super- $B M S_{3}$ algebra in the flat limit $\ell \rightarrow \infty$. An alternative way to obtain a $\mathcal{N}=2$ super- $B M S_{3}$ algebra in presence of R-symmetry generators $\mathcal{R}_{m}$ has been presented in [51] by expanding a $\mathcal{N}=2$ super-Virasoro algebra. The $\mathcal{N}=2$ super- $B M S_{3}$ algebra can also be recovered from the $\mathcal{N}=4$ super- $B M S_{3}$ appearing in [68] after setting some fermionic generators to zero. On the other hand, a "despotic" contraction of the $\mathcal{N}=(2,2)$ superconformal algebra reproduces an inequivalent $\mathcal{N}=2$ super- $B M S_{3}$ algebra [44].

\section{New examples}

4.1 Supersymmetric extension of the asymptotic algebra of the Maxwell gravity theory

In this section, we explore the supersymmetry extension of a particular infinite-dimensional algebra introduced in [50]. Such infinite-dimensional algebra results to describe the asymptotic symmetry of the three-dimensional ChernSimons gravity theory invariant under the Maxwell algebra [17]. In particular, as was shown in [17], the new infinitedimensional algebra is obtained by studying solutions of the Maxwell CS gravity theory with null boundary in the BMS gauge. Similarly to the finite subalgebra, given by the Maxwell algebra, the asymptotic symmetry contains an additional Abelian generator $\mathcal{Z}_{m}$. The presence of this additional generator modifies the $B M S_{3}$ algebra to an extension and deformation of such algebra introduced first as an expansion of the Virasoro algebra in [50]. Appendix A contains a brief review of the Maxwell gravity theory and its asymptotic symmetry.

The supersymmetric version of the extended and deformed $B M S_{3}$ algebra is unknown. Although the $\mathcal{N}$-extended CS supergravity theory based on a $\mathcal{N}$-extended Maxwell superalgebra has been studied in [53,73], the asymptotic structure 
remains unexplored. In what follows, we extend the results obtained in [50] to incorporate supersymmetry. In particular, we present diverse $\mathcal{N}$-extended supersymmetric versions of the asymptotic symmetry of the Maxwell CS gravity theory [17] by expanding the $\mathcal{N}$-extended super Virasoro algebra. For this purpose, we shall consider $S_{E}^{(4)}$ as the relevant semigroup. The election of the semigroup is not arbitrary and has two origins. First, as was shown in [50,53], the family $S_{E}^{(n)}$ of semigroups has been used to obtain the Maxwell (super)algebras from (super) Lorentz algebra. In particular, the Maxwell algebra belongs to a family of Maxwell like algebras denoted by $\mathfrak{B}_{k}$ where $k=4$ reproduces the Maxwell algebra. Such family can be obtained as a $S_{E}^{k-2}$-expansion [74]. Second, as was discussed at the bosonic level in [50], the semigroups used to relate finite algebras can also be used to relate their respective infinite-dimensional enhancements. For instance, as was shown in [50], the Maxwell algebra can be obtained as a $S_{E}^{(2)}$-expansion of the Lorentz algebra. Interestingly, the same semigroup is used in [50] to relate the respective infinite-dimensional algebras of the Maxwell and Lorentz one. Then it seems natural to choose $S_{E}^{(4)}$ as the relevant semigroup for our task since the Maxwell superalgebra can be obtained as a $S_{E}^{(4)}$-expansion of the super-Lorentz algebra [53].

We conjecture that the new infinite-dimensional superalgebras obtained here would correspond to the asymptotic symmetries of three-dimensional CS supergravity theories invariant under the $\mathcal{N}$-extended Maxwell superalgebra. The explicit obtention of such infinite-dimensional superalgebras by imposing suitable boundary conditions would be explored in a future work.

\subsubsection{Minimal deformed super- $\widetilde{B M S_{3}}$ algebra}

Let us consider the $S$-expansion of the super-Virasoro algebra (2.7) with $S_{E}^{(4)} \Im\left\{\lambda_{0}, \lambda_{1}, \lambda_{2}, \lambda_{3}, \lambda_{4}, \lambda_{5}\right\}$ as the relevant finite semigroup whose element satisfy the following multiplication law

$$
\begin{array}{l|llllll}
\lambda_{5} & \lambda_{5} & \lambda_{5} & \lambda_{5} & \lambda_{5} & \lambda_{5} & \lambda_{5} \\
\lambda_{4} & \lambda_{4} & \lambda_{5} & \lambda_{5} & \lambda_{5} & \lambda_{5} & \lambda_{5} \\
\lambda_{3} & \lambda_{3} & \lambda_{4} & \lambda_{5} & \lambda_{5} & \lambda_{5} & \lambda_{5} \\
\lambda_{2} & \lambda_{2} & \lambda_{3} & \lambda_{4} & \lambda_{5} & \lambda_{5} & \lambda_{5} \\
\lambda_{1} & \lambda_{1} & \lambda_{2} & \lambda_{3} & \lambda_{4} & \lambda_{5} & \lambda_{5} \\
\lambda_{0} & \lambda_{0} & \lambda_{1} & \lambda_{2} & \lambda_{3} & \lambda_{4} & \lambda_{5} \\
\hline & \lambda_{0} & \lambda_{1} & \lambda_{2} & \lambda_{3} & \lambda_{4} & \lambda_{5}
\end{array}
$$

with $\lambda_{5}=0_{S}$ being the zero element of the semigroup. Let $S_{E}^{(4)}=S_{0} \cup S_{1}$, with

$$
\begin{aligned}
& S_{0}=\left\{\lambda_{0}, \lambda_{2}, \lambda_{4}, \lambda_{5}\right\}, \\
& S_{1}=\left\{\lambda_{1}, \lambda_{3}, \lambda_{5}\right\},
\end{aligned}
$$

be the resonant subset decomposition. One can see that such decomposition has the same structure than the super-Virasoro subspaces (2.9),

$S_{0} \cdot S_{0} \subset S_{0}$,

$S_{0} \cdot S_{1} \subset S_{1}$,

$S_{1} \cdot S_{1} \subset S_{0}$.

A supersymmetric extension of the deformed $\widetilde{B M S}_{3}$ algebra (A.3) is obtained by considering a resonant subalgebra of $S_{E}^{(4)} \times \mathfrak{s v i r}$ and performing a $0_{S}$-reduction. Denoting the generators (2.11) and the central charges (2.12) of the corresponding $S$-expanded superalgebra as

$$
\begin{aligned}
& \mathcal{J}_{m}=\lambda_{0} \ell_{m}, \quad c_{1}=\lambda_{0} c, \\
& \ell \mathcal{P}_{m}=\lambda_{2} \ell_{m}, \quad \ell c_{2}=\lambda_{2} c, \\
& \ell^{2} \mathcal{Z}_{m}=\lambda_{4} \ell_{m}, \quad \ell^{2} c_{3}=\lambda_{4} c, \\
& \ell^{1 / 2} \mathcal{G}_{r}=\lambda_{1} \mathcal{Q}_{r}, \quad \ell^{3 / 2} \mathcal{H}_{r}=\lambda_{3} \mathcal{Q}_{r},
\end{aligned}
$$

the $0_{S}$-reduced and resonant $S_{E}^{(4)}$-expanded superalgebra satisfies the following non-vanishing (anti-)commutation relations:

$$
\begin{aligned}
& {\left[\mathcal{J}_{m}, \mathcal{J}_{n}\right]=(m-n) \mathcal{J}_{m+n}+\frac{c_{1}}{12} m\left(m^{2}-1\right) \delta_{m+n, 0}} \\
& {\left[\mathcal{J}_{m}, \mathcal{P}_{n}\right]=(m-n) \mathcal{P}_{m+n}+\frac{c_{2}}{12} m\left(m^{2}-1\right) \delta_{m+n, 0}} \\
& {\left[\mathcal{P}_{m}, \mathcal{P}_{n}\right]=(m-n) \mathcal{Z}_{m+n}+\frac{c_{3}}{12} m\left(m^{2}-1\right) \delta_{m+n, 0},} \\
& {\left[\mathcal{J}_{m}, \mathcal{Z}_{n}\right]=(m-n) \mathcal{Z}_{m+n}+\frac{c_{3}}{12} m\left(m^{2}-1\right) \delta_{m+n, 0},} \\
& {\left[\mathcal{J}_{m}, \mathcal{G}_{r}\right]=\left(\frac{m}{2}-r\right) \mathcal{G}_{m+r}, \quad\left[\mathcal{P}_{m}, \mathcal{G}_{r}\right]=\left(\frac{m}{2}-r\right) \mathcal{H}_{m+r}} \\
& {\left[\mathcal{J}_{m}, \mathcal{H}_{r}\right]=\left(\frac{m}{2}-r\right) \mathcal{H}_{m+r},} \\
& \left\{\mathcal{G}_{r}, \mathcal{G}_{s}\right\}=\mathcal{P}_{r+s}+\frac{c_{2}}{6}\left(r^{2}-\frac{1}{4}\right) \delta_{r+s, 0} \\
& \left\{\mathcal{G}_{r}, \mathcal{H}_{s}\right\}=\mathcal{Z}_{r+s}+\frac{c_{3}}{6}\left(r^{2}-\frac{1}{4}\right) \delta_{r+s, 0} .
\end{aligned}
$$

Such infinite-dimensional superalgebra is the minimal supersymmetric extension of the so-called deformed $\widetilde{B M S}_{3}$ algebra (A.3) and appears by combining the semigroup multiplication law (4.1) with the original (anti-)commutators of the super-Virasoro algebra (2.7). Such supersymmetric extension is characterized by the introduction of an additional spinor charge $\mathcal{H}_{r}$ whose presence assures the Jacobi identity $(\mathcal{P}, \mathcal{G}, \mathcal{G})$. Analogously to its bosonic version, the deformed super- $\widehat{B M S}_{3}$ algebra contains a finite subalgebra spanned by the generators $\mathcal{J}_{0}, \mathcal{J}_{1}, \mathcal{J}_{-1}, \mathcal{P}_{0}, \mathcal{P}_{1}, \mathcal{P}_{-1}, \mathcal{Z}_{0}, \mathcal{Z}_{1}, \mathcal{Z}_{-1}, \mathcal{G}_{\frac{1}{2}}$, $\mathcal{G}_{-\frac{1}{2}}$ and $\mathcal{H}_{\frac{1}{2}}, \mathcal{H}_{-\frac{1}{2}}$ which are related to the minimal Maxwell superalgebra 
$\mathcal{J}_{-1}=-\sqrt{2} J_{0}, \quad \mathcal{J}_{1}=\sqrt{2} J_{1}, \quad \mathcal{J}_{0}=J_{2}$,

$\mathcal{P}_{-1}=-\sqrt{2} P_{0}, \mathcal{P}_{1}=\sqrt{2} P_{1}, \quad \mathcal{P}_{0}=P_{2}$,

$\mathcal{Z}_{-1}=-\sqrt{2} Z_{0}, \mathcal{Z}_{1}=\sqrt{2} Z_{1}, \quad \mathcal{Z}_{0}=Z_{2}$

$\mathcal{G}_{-\frac{1}{2}}=\sqrt{2} Q_{+}, \quad \mathcal{G}_{\frac{1}{2}}=\sqrt{2} Q_{-}$,

$\mathcal{H}_{-\frac{1}{2}}=\sqrt{2} \Sigma_{+}, \mathcal{H}_{\frac{1}{2}}=\sqrt{2} \Sigma_{-}$.

This means that the minimal deformed super- $\widetilde{B M S_{3}}$ algebra (4.5) obtained here corresponds to an infinite-dimensional lift of the minimal Maxwell superalgebra in the very same way as the deformed $\widetilde{B M S}$ algebra is an infinitedimensional lift of the Maxwell algebra. In particular, the super-Maxwell generators satisfy the following nonvanishing (anti-)commutators:

$$
\begin{aligned}
{\left[J_{a}, J_{b}\right] } & =\epsilon_{a b c} J^{c}, \quad\left[J_{a}, P_{b}\right]=\epsilon_{a b c} P^{c}, \\
{\left[J_{a}, Z_{b}\right] } & =\epsilon_{a b c} Z^{c}, \quad\left[P_{a}, P_{b}\right]=\epsilon_{a b c} Z^{c}, \\
{\left[J_{a}, Q_{\alpha}\right] } & =\frac{1}{2}\left(\Gamma_{a}\right)_{\alpha}^{\beta} Q_{\beta}, \\
{\left[J_{a}, \Sigma_{\alpha}\right] } & =\frac{1}{2}\left(\Gamma_{a}\right)_{\alpha}^{\beta} \Sigma_{\beta}, \\
{\left[P_{a}, Q_{\alpha}\right] } & =\frac{1}{2}\left(\Gamma_{a}\right)_{\alpha}^{\beta} \Sigma_{\beta}, \\
\left\{Q_{\alpha}, Q_{\beta}\right\} & =\frac{1}{2}\left(C \Gamma^{a}\right)_{\alpha \beta} P_{a}, \\
\left\{Q_{\alpha}, \Sigma_{\beta}\right\} & =\frac{1}{2}\left(C \Gamma^{a}\right)_{\alpha \beta} Z_{a} .
\end{aligned}
$$

The supersymmetrization of the Maxwell algebra is not unique and have been studied by diverse authors [75-86]. However as was discussed in [53], the superalgebra spanned by $\left\{J_{a}, P_{a}, Z_{a}, Q_{\alpha}, \Sigma_{\alpha}\right\}$ is the minimal supersymmetric extension of the Maxwell algebra allowing to define a consistent supergravity action in three spacetime dimensions. Let us note that the presence of a second Abelian spinorial charge has already been discussed in the context of superstring theory [87] and $D=11$ supergravity [88].

As an ending remark, it is worth it to mention that the three-dimensional minimal Maxwell supergravity theory constructed in [53] has directly been obtained through the semigroup expansion using $S_{E}^{(4)}$ as the relevant finite semigroup. As we have discussed in Sect. 2, the semigroup used to obtain a finite (super)algebra from a finite one can also be used to relate their respective infinite-dimensional enhancements. Such particular feature is the main reason behind our election of semigroups.

\subsection{2 $\mathcal{N}=2$ deformed super $-\widetilde{B M S} 3$ algebra}

The extension to $\mathcal{N}=(2,0)$ of the deformed super- $B M S_{3}$ algebra requires to consider the $\mathcal{N}=2$ super-Virasoro algebra as the starting point. Interestingly, the $\mathcal{N}=2$ deformed super- $\widehat{B M S} S_{3}$ algebra obtained through the semigroup expansion procedure corresponds to the supersymmetric extension of the deformed $\widetilde{B M S} S_{3}$ algebra (A.3) endowed with three $\hat{\mathfrak{u}}(1)$ current algebra.

The $\mathcal{N}=2$ super-Virasoro algebra, which we shall denote as $\mathfrak{s v i r}(2)$, is characterized by the presence of an R-symmetry generator $\mathcal{R}_{m}$ and its (anti-)commutators are given by

$$
\begin{aligned}
{\left[\ell_{m}, \ell_{n}\right] } & =(m-n) \ell_{m+n}+\frac{c}{12} m\left(m^{2}-1\right) \delta_{m+n, 0}, \\
{\left[\ell_{m}, \mathcal{Q}_{r}^{i}\right]=} & \left(\frac{m}{2}-r\right) \mathcal{Q}_{m+r}^{i}, \\
{\left[\ell_{m}, \mathcal{R}_{n}\right]=} & -n \mathcal{R}_{m+n}, \\
{\left[\mathcal{R}_{m}, \mathcal{R}_{n}\right]=} & \frac{c}{3} m \delta_{m+n, 0}, \\
{\left[\mathcal{Q}_{r}^{i}, \mathcal{R}_{m}\right]=} & \epsilon^{i j} \mathcal{Q}_{m+r}^{j}, \\
\left\{\mathcal{Q}_{r}^{i}, \mathcal{Q}_{s}^{j}\right\}= & \delta^{i j}\left[\ell_{r+s}+\frac{c}{6}\left(r^{2}-\frac{1}{4}\right) \delta_{r+s, 0}\right] \\
& -2 \epsilon^{i j}(r-s) \mathcal{R}_{r+s},
\end{aligned}
$$

One can notice that the $\mathcal{N}=2$ super-Virasoro algebra can be decomposed in a bosonic subspace $V_{0}=\left\{\ell_{m}, \mathcal{R}_{m}, c\right\}$ and a fermionic subspace $V_{1}=\left\{\mathcal{Q}_{r}^{i}\right\}$ such that

$\mathfrak{s v i r}_{(2)}=V_{0} \oplus V_{1}$,

where they satisfy a graded Lie algebra (2.9).

On the other hand, let us consider $S_{E}^{(4)}=\left\{\lambda_{0}, \lambda_{1}, \lambda_{2}, \lambda_{3}, \lambda_{4}\right.$, $\left.\lambda_{5}\right\}$ as the relevant finite semigroup whose elements satisfy the multiplication law (4.1) and $\lambda_{5}=0_{S}$ being the zero element of the semigroup. Let $S_{E}^{(4)}=S_{0} \cup S_{1}$ with

$S_{0}=\left\{\lambda_{0}, \lambda_{2}, \lambda_{4}, \lambda_{5}\right\}$,

$S_{1}=\left\{\lambda_{1}, \lambda_{3}, \lambda_{5}\right\}$,

be the subset decomposition which is resonant with the subspace decomposition (4.9). Then, an $S$-expanded superalgebra generated by the set $\left\{\mathcal{J}_{m}, \mathcal{P}_{m}, \mathcal{Z}_{m}, \mathrm{~T}_{m}, \mathrm{~B}_{m}, \mathrm{Z}_{m}, \mathcal{G}_{r}^{i}, \mathcal{H}_{r}^{i}\right.$, $\left.c_{1}, c_{2}, c_{3}\right\}$ can be obtained by considering a $0_{S}$-reduced resonant subalgebra of $S_{E}^{(4)} \times \mathfrak{s v i r}_{(2)}$. In particular, the expanded generators and central charges are related to the $\mathcal{N}=2$ superVirasoro ones through

$$
\begin{aligned}
& \mathcal{J}_{m}=\lambda_{0} \ell_{m}, \quad c_{1}=\lambda_{0} c, \\
& \ell \mathcal{P}_{m}=\lambda_{2} \ell_{m}, \quad \ell c_{2}=\lambda_{2} c, \\
& \ell^{2} \mathcal{Z}_{m}=\lambda_{4} \ell_{m}, \quad \ell^{2} c_{3}=\lambda_{4} c, \\
& \mathrm{~T}_{m}=\lambda_{0} \mathcal{R}_{m}, \quad \ell \mathrm{B}_{m}=\lambda_{2} \mathcal{R}_{m}, \\
& \ell^{2} \mathrm{Z}_{m}=\lambda_{4} \mathcal{R}_{m}, \\
& \ell^{1 / 2} \mathcal{G}_{r}=\lambda_{1} \mathcal{Q}_{r}, \ell^{3 / 2} \mathcal{H}_{r}=\lambda_{3} \mathcal{Q}_{r} .
\end{aligned}
$$

Such generators satisfy an $\mathcal{N}=2$ supersymmetric extension of the deformed $\widehat{B M S}_{3}$ algebra whose (anti-)commutation relations are directly obtained by combining the multiplication law of the semigroup (4.1) and the original (anti-) commutatorsof the $\mathcal{N}=2$ super-Virasoro algebra (4.8). In fact, 
the (anti-)commutators of the $S$-expanded superalgebra are given by

$$
\begin{aligned}
& {\left[\mathcal{J}_{m}, \mathcal{J}_{n}\right]=(m-n) \mathcal{J}_{m+n}+\frac{c_{1}}{12} m\left(m^{2}-1\right) \delta_{m+n, 0},} \\
& {\left[\mathcal{J}_{m}, \mathcal{P}_{n}\right]=(m-n) \mathcal{P}_{m+n}+\frac{c_{2}}{12} m\left(m^{2}-1\right) \delta_{m+n, 0},} \\
& {\left[\mathcal{P}_{m}, \mathcal{P}_{n}\right]=(m-n) \mathcal{Z}_{m+n}+\frac{c_{3}}{12} m\left(m^{2}-1\right) \delta_{m+n, 0},} \\
& {\left[\mathcal{J}_{m}, \mathcal{Z}_{n}\right]=(m-n) \mathcal{Z}_{m+n}+\frac{c_{3}}{12} m\left(m^{2}-1\right) \delta_{m+n, 0},} \\
& {\left[\mathcal{J}_{m}, \mathrm{~T}_{n}\right]=-n \mathrm{~T}_{m+n}, \quad\left[\mathcal{P}_{m}, \mathrm{~T}_{n}\right]=-n \mathrm{~B}_{m+n},} \\
& {\left[\mathcal{Z}_{m}, \mathrm{~T}_{n}\right]=-n \mathrm{Z}_{m+n}, \quad\left[\mathcal{J}_{m}, \mathrm{~B}_{n}\right]=-n \mathrm{~B}_{m+n},} \\
& {\left[\mathcal{P}_{m}, \mathrm{~B}_{n}\right]=-n \mathrm{Z}_{m+n}, \quad\left[\mathcal{J}_{m}, \mathrm{Z}_{n}\right]=-n \mathrm{Z}_{m+n},} \\
& {\left[\mathrm{~T}_{m}, \mathrm{~T}_{n}\right]=\frac{c_{1}}{3} m \delta_{m+n, 0}, \quad\left[\mathrm{~T}_{m}, \mathrm{~B}_{n}\right]=\frac{c_{2}}{3} m \delta_{m+n, 0},} \\
& {\left[\mathrm{~T}_{m}, \mathrm{Z}_{n}\right]=\frac{c_{3}}{3} m \delta_{m+n, 0}, \quad\left[\mathrm{~B}_{m}, \mathrm{~B}_{n}\right]=\frac{c_{3}}{3} m \delta_{m+n, 0},} \\
& {\left[\mathcal{J}_{m}, \mathcal{G}_{r}^{i}\right]=\left(\frac{m}{2}-r\right) \mathcal{G}_{m+r}^{i}, \quad\left[\mathcal{P}_{m}, \mathcal{G}_{r}^{i}\right]} \\
& =\left(\frac{m}{2}-r\right) \mathcal{H}_{m+r}^{i}, \\
& {\left[\mathcal{J}_{m}, \mathcal{H}_{r}^{i}\right]=\left(\frac{m}{2}-r\right) \mathcal{H}_{m+r}^{i},} \\
& {\left[\mathcal{G}_{r}^{i}, \mathrm{~T}_{m}\right]=\epsilon^{i j} \mathcal{G}_{m+r}^{j}, \quad\left[\mathcal{G}_{r}^{i}, \mathrm{~B}_{m}\right]=\epsilon^{i j} \mathcal{H}_{m+r}^{j},} \\
& {\left[\mathcal{H}_{r}^{i}, \mathrm{~T}_{m}\right]=\epsilon^{i j} \mathcal{H}_{m+r}^{j},} \\
& \left\{\mathcal{G}_{r}^{i}, \mathcal{G}_{s}^{j}\right\}=\delta^{i j}\left[\mathcal{P}_{r+s}+\frac{c_{2}}{6}\left(r^{2}-\frac{1}{4}\right) \delta_{r+s, 0}\right] \\
& -2 \epsilon^{i j}(r-s) \mathrm{B}_{r+s}, \\
& \left\{\mathcal{G}_{r}^{i}, \mathcal{H}_{s}^{j}\right\}=\delta^{i j}\left[\mathcal{Z}_{r+s}+\frac{c_{3}}{6}\left(r^{2}-\frac{1}{4}\right) \delta_{r+s, 0}\right] \\
& -2 \epsilon^{i j}(r-s) \mathrm{Z}_{r+s} .
\end{aligned}
$$

Such $\mathcal{N}=2$ deformed super- $\widetilde{B M S} S_{3}$ algebra differs from the $\mathcal{N}=1$ one by the presence of additional bosonic generators. In particular, one can see that the anticommutator of the supercharges $\mathcal{G}_{r}^{i}$ closes to a combination of $\mathcal{P}, \mathrm{B}$ and a central charge $c_{2}$. On the other hand, the anticommutator of the supercharges $\mathcal{G}_{r}^{i}$ and $\mathcal{H}_{r}^{i}$ closes to a combination of $\mathcal{Z}$, Zand a central charge $c_{3}$. The present superalgebra can be seen as the $\mathcal{N}=2$ supersymmetric extension of deformed $\widehat{B M S_{3}}$ algebra endowed with $\hat{\mathfrak{u}}(1) \times \hat{\mathfrak{u}}(1) \times \hat{\mathfrak{u}}(1)$ current algebra. This can be seen more clearly by the fact that the infinite-dimensional superalgebra obtained here can alternatively be recovered as an Inönü-Wigner contraction of three copies of a Virasoro algebra, two of which augmented by supersymmetry, endowed with an affine $\hat{\mathfrak{u}}$ (1) current algebra. In particular, the $\hat{\mathfrak{u}}$ (1) current generators $\left\{\mathfrak{k}_{n}, \overline{\mathfrak{k}}_{n}, \tilde{\mathfrak{k}}_{n}\right\}$ are related to the $\mathcal{N}=2$ deformed super- $\widetilde{B M S_{3}}$ ones through the following redefinitions

$$
\begin{aligned}
\mathrm{T}_{m} & =\mathfrak{k}_{m}+\overline{\mathfrak{k}}_{-m}+\tilde{\mathfrak{k}}_{-m}, \quad \mathrm{~B}_{m}=\epsilon\left(\mathfrak{k}_{m}-\overline{\mathfrak{k}}_{-m}\right), \quad \mathrm{Z}_{m} \\
& =\epsilon^{2}\left(\mathfrak{k}_{m}+\overline{\mathfrak{k}}_{-m}\right),
\end{aligned}
$$

where the limit $\epsilon \rightarrow 0$ reproduces the $\mathcal{N}=2$ deformed super- $\widetilde{B M S} S_{3}$ algebra presented here. The presence of $\hat{\mathfrak{u}}(1)$ current generators in asymptotic symmetries is not new and has already be considered in $B M S_{3}$ algebra $[13,14,89]$ and $\mathcal{N}=2$ super- $B M S_{3}$ algebra [51].

One can note that the central extension of the $\mathcal{N}=2$ Maxwell superalgebra endowed with $\mathfrak{s o}(2)$ internal symmetry generators [73] appears as a finite subalgebra of the $\mathcal{N}=2$ deformed super- $B M S_{3}$ algebra. Such subalgebra is spanned by the generators $\mathcal{J}_{0}, \mathcal{J}_{ \pm 1}, \mathcal{P}_{0}, \mathcal{P}_{ \pm 1}, \mathcal{Z}_{0}, \mathcal{Z}_{ \pm 1}, \mathrm{~T}_{0}, \mathrm{~B}_{0}, \mathrm{Z}_{0}$, $\mathcal{G}_{ \pm \frac{1}{2}}$ and $\mathcal{H}_{ \pm \frac{1}{2}}$ which are related to the $\mathcal{N}=2$ Maxwell superalgebra $\left\{J_{a}, P_{a}, Z_{a}, T, B, Z, Q, \Sigma\right\}$ as follows

$$
\begin{array}{lll}
\mathcal{J}_{-1}=-\sqrt{2} J_{0}, & \mathcal{J}_{1}=\sqrt{2} J_{1}, & \mathcal{J}_{0}=J_{2}, \\
\mathcal{P}_{-1}=-\sqrt{2} P_{0}, & \mathcal{P}_{1}=\sqrt{2} P_{1}, & \mathcal{P}_{0}=P_{2}, \\
\mathcal{Z}_{-1}=-\sqrt{2} Z_{0}, & \mathcal{Z}_{1}=\sqrt{2} Z_{1}, & \mathcal{Z}_{0}=Z_{2}, \\
\mathrm{~T}_{0}=-T, & \mathrm{~B}_{0}=-B, & \mathrm{Z}_{0}=-Z, \\
\mathcal{G}_{-\frac{1}{2}}=\sqrt{2} Q_{+}, & \mathcal{G}_{\frac{1}{2}}=\sqrt{2} Q_{-}, \\
\mathcal{H}_{-\frac{1}{2}}=\sqrt{2} \Sigma_{+}, & \mathcal{H}_{\frac{1}{2}}=\sqrt{2} \Sigma_{-} .
\end{array}
$$

Remarkably, the central extension of the $\mathcal{N}=2$ Maxwell superalgebra endowed with $\mathfrak{s o}(2)$ internal symmetry generators allows to define an invariant non-degenerate innerproduct which provides us with a consistent three-dimensional supergravity action [73]. Interestingly, analogously to the results presented here, the $\mathcal{N}=2$ Maxwell supergravity theory can alternatively be obtained by considering an $S$ expansion of the $\mathcal{N}=2$ super Lorentz algebra using the same semigroup $S_{E}^{(4)}$.

\subsection{3 $\mathcal{N}=4$ deformed super $-\widetilde{B M S}$ algebra}

Here we extend our construction to the obtention of the $\mathcal{N}=(4,0)$ deformed super- $\widehat{B M S_{3}}$ algebra by considering an $S_{E}^{(4)}$-expansion of the $\mathcal{N}=4$ super-Virasoro algebra. The infinite-dimensional superalgebra obtained corresponds to the supersymmetric extension of the deformed $\widehat{B M S}_{3}$ algebra (A.3) endowed with three $\mathfrak{s u}$ (2) current algebra.

Let us consider the $\mathcal{N}=4$ super-Virasoro algebra whose (anti-)commutation relations are given by [90]

$$
\begin{aligned}
{\left[\ell_{m}, \ell_{n}\right] } & =(m-n) \ell_{m+n}+\frac{c}{12} m\left(m^{2}-1\right) \delta_{m+n, 0}, \\
{\left[\ell_{m}, \mathcal{Q}_{r}^{i, \pm}\right] } & =\left(\frac{m}{2}-r\right) \mathcal{Q}_{m+r}^{i, \pm}, \\
{\left[\ell_{m}, \mathcal{R}_{n}^{a}\right] } & =-n \mathcal{R}_{m+n}^{a}, \\
{\left[\mathcal{R}_{m}^{a}, \mathcal{R}_{n}^{b}\right] } & =i \epsilon^{a b c} \mathcal{R}_{m+n}^{c}+\frac{c}{12} m \delta^{a b} \delta_{m+n, 0}, \\
{\left[\mathcal{R}_{m}^{a}, \mathcal{Q}_{r}^{i,+}\right] } & =-\frac{1}{2}\left(\sigma^{a}\right)_{j}^{i} \mathcal{Q}_{m+r}^{j,+}, \quad\left[\mathcal{R}_{m}^{a}, \mathcal{Q}_{r}^{i,-}\right]
\end{aligned}
$$




$$
\begin{aligned}
= & \frac{1}{2}\left(\bar{\sigma}^{a}\right)_{j}^{i} \mathcal{Q}_{m+r}^{j,-}, \\
\left\{\mathcal{Q}_{r}^{i,+}, \mathcal{Q}_{s}^{j,-}\right\}= & \delta^{i j}\left[\ell_{r+s}+\frac{c}{6}\left(r^{2}-\frac{1}{4}\right) \delta_{r+s, 0}\right] \\
& -(r-s)\left(\sigma^{a}\right)_{i j} \mathcal{R}_{r+s}^{a},
\end{aligned}
$$

where $\sigma_{i j}^{a}=\sigma_{j i}^{a}$ are the Pauli matrices, $i, j=1,2$ and $a, b, c=1,2,3$. As the $\mathcal{N}=1$ and $\mathcal{N}=2$ version, the $\mathcal{N}=4$ super-Virasoro algebra can be written as the direct sum of a bosonic subspace $V_{0}=\left\{\ell_{m}, \mathcal{R}_{m}^{a}, c\right\}$ and a fermionic one $V_{1}=\left\{Q_{r}^{i, \pm}\right\}$.

Let $S_{E}^{(4)}=\left\{\lambda_{0}, \lambda_{1}, \lambda_{2}, \lambda_{3}, \lambda_{4}, \lambda_{5}\right\}$ be the relevant Abelian semigroup whose elements satisfy (4.1). Then, by considering the resonant decomposition (4.10) and applying a resonant $0_{S}$-reduction $S_{E}^{(4)}$-expansion of the $\mathcal{N}=4$ superVirasoro algebra we find an expanded algebra spanned by the set of generators:

$$
\left\{\mathcal{J}_{m}, \mathcal{P}_{m}, \mathcal{Z}_{m}, \mathrm{~T}_{m}^{a}, \mathrm{~B}_{m}^{a}, \mathrm{Z}_{m}^{a}, \mathcal{G}_{r}^{i, \pm}, \mathcal{H}_{r}^{i, \pm}, c_{1}, c_{2}, c_{3}\right\}
$$

The expanded generators and central charges can be written in terms of the $\mathcal{N}=4$ super-Virasoro ones through the semigroup elements as

$$
\begin{array}{ll}
\mathcal{J}_{m}=\lambda_{0} \ell_{m}, & c_{1}=\lambda_{0} c, \\
\ell \mathcal{P}_{m}=\lambda_{2} \ell_{m}, & \ell c_{2}=\lambda_{2} c, \\
\ell^{2} \mathcal{Z}_{m}=\lambda_{4} \ell_{m}, & \ell^{2} c_{3}=\lambda_{4} c, \\
\mathrm{~T}_{m}^{a}=\lambda_{0} \mathcal{R}_{m}^{a}, & \ell \mathrm{B}_{m}^{a}=\lambda_{2} \mathcal{R}_{m}^{a}, \\
\ell^{2} \mathrm{Z}_{m}^{a}=\lambda_{4} \mathcal{R}_{m}^{a}, & \\
\ell^{1 / 2} \mathcal{G}_{r}^{i, \pm}=\lambda_{1} \mathcal{Q}_{r}^{i, \pm}, \ell^{3 / 2} \mathcal{H}_{r}^{i, \pm}=\lambda_{3} \mathcal{Q}_{r}^{i, \pm} .
\end{array}
$$

Using the semigroup multiplication law (4.1) and the original (anti-)commutators of the $\mathcal{N}=4$ super-Virasoro algebra (4.17) one can see that the expanded generators satisfy a $\mathcal{N}=$ 4 deformed super- $\widetilde{B M S}_{3}$ algebra whose (anti-)commutation relations are given by (A.3) and

$$
\begin{aligned}
{\left[\mathcal{J}_{m}, \mathrm{~T}_{n}^{a}\right] } & =-n \mathrm{~T}_{m+n}^{a}, \quad\left[\mathcal{P}_{m}, \mathrm{~T}_{n}^{a}\right]=-n \mathrm{~B}_{m+n}^{a}, \\
{\left[\mathcal{Z}_{m}, \mathrm{~T}_{n}^{a}\right] } & =-n \mathrm{Z}_{m+n}^{a}, \quad\left[\mathcal{J}_{m}, \mathrm{~B}_{n}^{a}\right]=-n \mathrm{~B}_{m+n}^{a}, \\
{\left[\mathcal{P}_{m}, \mathrm{~B}_{n}^{a}\right] } & =-n \mathrm{Z}_{m+n}^{a}, \quad\left[\mathcal{J}_{m}, \mathrm{Z}_{n}^{a}\right]=-n \mathrm{Z}_{m+n}^{a}, \\
{\left[\mathrm{~T}_{m}^{a}, \mathrm{~T}_{n}^{b}\right] } & =i \epsilon^{a b c} \mathrm{~T}_{m+n}^{c}+\frac{c_{1}}{12} m \delta^{a b} \delta_{m+n, 0}, \\
{\left[\mathrm{~T}_{m}^{a}, \mathrm{~B}_{n}^{b}\right] } & =i \epsilon^{a b c} \mathrm{~B}_{m+n}^{c}+\frac{c_{2}}{12} m \delta^{a b} \delta_{m+n, 0}, \\
{\left[\mathrm{~T}_{m}^{a}, \mathrm{Z}_{n}^{b}\right] } & =i \epsilon^{a b c} \mathrm{Z}_{m+n}^{c}+\frac{c_{3}}{12} m \delta^{a b} \delta_{m+n, 0}, \\
{\left[\mathrm{~B}_{m}^{a}, \mathrm{~B}_{n}^{b}\right] } & =i \epsilon^{a b c} \mathrm{Z}_{m+n}^{c}+\frac{c_{3}}{12} m \delta^{a b} \delta_{m+n, 0}, \\
{\left[\mathcal{J}_{m}, \mathcal{G}_{r}^{i, \pm}\right] } & =\left(\frac{m}{2}-r\right) \mathcal{G}_{m+r}^{i, \pm}, \quad\left[\mathcal{P}_{m}, \mathcal{G}_{r}^{i, \pm}\right] \\
& =\left(\frac{m}{2}-r\right) \mathcal{H}_{m+r}^{i, \pm}, \\
{\left[\mathcal{J}_{m}, \mathcal{H}_{r}^{i, \pm}\right] } & =\left(\frac{m}{2}-r\right) \mathcal{H}_{m+r}^{i, \pm},
\end{aligned}
$$

$$
\begin{aligned}
{\left[\mathrm{T}_{m}^{a}, \mathcal{G}_{r}^{i,+}\right]=} & -\frac{1}{2}\left(\sigma^{a}\right)_{j}^{i} \mathcal{G}_{m+r}^{j,+}, \quad\left[\mathrm{T}_{m}^{a}, \mathcal{G}_{r}^{i,-}\right] \\
= & \frac{1}{2}\left(\bar{\sigma}^{a}\right)_{j}^{i} \mathcal{G}_{m+r}^{j,-}, \\
{\left[\mathrm{B}_{m}^{a}, \mathcal{G}_{r}^{i,+}\right]=} & -\frac{1}{2}\left(\sigma^{a}\right)_{j}^{i} \mathcal{H}_{m+r}^{j,+}, \quad\left[\mathrm{B}_{m}^{a}, \mathcal{G}_{r}^{i,-}\right] \\
= & \frac{1}{2}\left(\bar{\sigma}^{a}\right)_{j}^{i} \mathcal{H}_{m+r}^{j,-}, \\
{\left[\mathrm{T}_{m}^{a}, \mathcal{H}_{r}^{i,+}\right]=} & -\frac{1}{2}\left(\sigma^{a}\right)_{j}^{i} \mathcal{H}_{m+r}^{j,+}, \quad\left[\mathrm{T}_{m}^{a}, \mathcal{H}_{r}^{i,-}\right] \\
= & \frac{1}{2}\left(\bar{\sigma}^{a}\right)_{j}^{i} \mathcal{H}_{m+r}^{j,-}, \\
\left\{\mathcal{G}_{r}^{i,+}, \mathcal{G}_{s}^{j,-}\right\}= & \delta^{i j}\left[\mathcal{P}_{r+s}+\frac{c_{2}}{6}\left(r^{2}-\frac{1}{4}\right) \delta_{r+s, 0}\right] \\
& -(r-s)\left(\sigma^{a}\right)_{i j} \mathrm{~B}_{r+s}^{a}, \\
\left\{\mathcal{G}_{r}^{i,+}, \mathcal{H}_{s}^{j,-}\right\}= & \delta^{i j}\left[\mathcal{Z}_{r+s}+\frac{c_{3}}{6}\left(r^{2}-\frac{1}{4}\right) \delta_{r+s, 0}\right] \\
& -(r-s)\left(\sigma^{a}\right)_{i j} z_{r+s}^{a} .
\end{aligned}
$$

Such infinite-dimensional superalgebra corresponds to the $\mathcal{N}=4$ supersymmetric extension of the deformed $\widehat{B M S_{3}}$ algebra endowed with $\mathfrak{s u}$ (2) current algebra spanned by $\mathfrak{k}_{m}^{a}$, $\overline{\mathfrak{k}}_{m}^{a}$ and $\tilde{\mathfrak{k}}_{m}^{a}$. The $\mathfrak{s u}(2)$ current generators are related to $\mathrm{T}_{m}^{a}$, $\mathrm{B}_{m}^{a}$ and $\mathrm{Z}_{m}^{a}$ through the following redefinitions:

$$
\begin{aligned}
\mathrm{T}_{m}^{a} & =\mathfrak{k}_{m}^{a}+\overline{\mathfrak{k}}_{-m}^{a}+\tilde{\mathfrak{k}}_{-m}^{a}, \quad \mathrm{~B}_{m}^{a}=\lim _{\epsilon \rightarrow 0} \epsilon\left(\mathfrak{k}_{m}^{a}-\overline{\mathfrak{k}}_{-m}^{a}\right), \quad \mathrm{Z}_{m}^{a} \\
& =\lim _{\epsilon \rightarrow 0} \epsilon^{2}\left(\mathfrak{k}_{m}^{a}+\overline{\mathfrak{k}}_{-m}^{a}\right),
\end{aligned}
$$

One can note that the presence of the $\mathcal{Z}_{m}, \mathcal{H}_{r}^{i, \pm}$ and $\mathrm{z}_{m}^{a}$ generators extends and deforms the $\mathcal{N}=4$ super- $B M S_{3}$ algebra presented in [51].

On the other hand, such infinite-dimensional superalgebra contains a finite subalgebra which corresponds to the central extension of the $\mathcal{N}=(4,0)$ Maxwell superalgebra endowed with internal symmetry generators [73]. Indeed, the set of generators $\left\{\mathcal{J}_{m}, \mathcal{P}_{m}, Z_{m}, \mathrm{~T}_{0}^{a}, \mathrm{~B}_{0}^{a}, \mathrm{z}_{0}^{a}, \mathcal{G}_{r}^{i, \pm}, \mathcal{H}_{r}^{i, \pm}\right\}$ with $m, n=0, \pm 1$ and $r= \pm \frac{1}{2}$ reproduces the $\mathcal{N}=4$ Maxwell superalgebra. It is worth it to mention that the $\mathcal{N}=4$ Maxwell superalgebra can also be obtained by applying an $S$-expansion to the $\mathcal{N}=4$ super Lorentz algebra considering the same semigroup $S_{E}^{(4)}$ used to obtain the $\mathcal{N}=4$ deformed super- $\widetilde{B M S} S_{3}$ algebra.

4.2 Supersymmetric extension of the asymptotic algebra of the $\mathfrak{s o}(2,2) \oplus \mathfrak{s o}(2,1)$ gravity theory

Let us now explore the supersymmetric extension of the asymptotic symmetry of the $\mathfrak{s o}(2,2) \oplus \mathfrak{s o}(2,1)$ CS gravity theory. As shown in [34], an explicit realisation of the asymptotic symmetry at null infinity turned out to be a semisimple enlargement of the $B M S_{3}$ algebra (see Appendix B for further details). Such infinite-dimensional algebra was 
first introduced in [50] as an $S$-expansion of the Virasoro algebra using the same semigroup $S_{\mathcal{M}}^{(2)}$ used for obtaining its finite subalgebra. Indeed, the AdS-Lorentz algebra can be obtained as a $S_{\mathcal{M}}^{(2)}$-expansion of the Lorentz algebra.

Here, we present diverse supersymmetric extensions of the enlarged $B M S_{3}$ algebra by $S$-expanding the $\mathcal{N}$-extended super-Virasoro algebra for $\mathcal{N}=1,2$ and 4 . To this purpose we shall use $S_{\mathcal{M}}^{(4)}$ as the relevant semigroup. This semigroup is characterized by the absence of zero elements which implies non-vanishing commutators in the expanded algebra. Furthermore this election is due to the fact that, as was discussed in [50], the semigroup relating two finite algebras can also be used to relate their respective infinite-dimensional algebras. Since the AdS-Lorentz superalgebra can be obtained as a Sexpansion of the Lorentz superalgebra using $S_{\mathcal{M}}^{(4)}$ as the semigroup, it seems then natural to apply the same semigroup $S_{\mathcal{M}}^{(4)}$ to the super Virasoro algebra in order to obtain the infinitedimensional enhancement of the AdS-Lorentz superalgebra. One could argue that the new infinite-dimensional superalgebras presented here would be the respective asymptotic symmetries of three-dimensional AdS-Lorentz CS supergravity theories.

\subsubsection{Minimal enlarged super-BMS $S_{3}$ algebra}

Let us consider the super-Virasoro algebra (2.7) as our starting algebra. Let $S_{\mathcal{M}}^{(4)}=\left\{\lambda_{0}, \lambda_{1}, \lambda_{2}, \lambda_{3}, \lambda_{4}\right\}$ be the relevant semigroup whose elements satisfy the following multiplication law

$$
\begin{array}{l|lllll}
\lambda_{4} & \lambda_{4} & \lambda_{1} & \lambda_{2} & \lambda_{3} & \lambda_{4} \\
\lambda_{3} & \lambda_{3} & \lambda_{4} & \lambda_{1} & \lambda_{2} & \lambda_{3} \\
\lambda_{2} & \lambda_{2} & \lambda_{3} & \lambda_{4} & \lambda_{1} & \lambda_{2} \\
\lambda_{1} & \lambda_{1} & \lambda_{2} & \lambda_{3} & \lambda_{4} & \lambda_{1} \\
\lambda_{0} & \lambda_{0} & \lambda_{1} & \lambda_{2} & \lambda_{3} & \lambda_{4} \\
\hline & \lambda_{0} & \lambda_{1} & \lambda_{2} & \lambda_{3} & \lambda_{4}
\end{array}
$$

One can notice that, unlike the $S_{E}$ semigroups, there is no zero element in the $S_{\mathcal{M}}$ family. The absence of zero element implies that there is no vanishing commutation relations in the expanded algebra.

Let us consider now a subset decomposition $S_{\mathcal{M}}^{(4)}=S_{0} \cup S_{1}$ with

$S_{0}=\left\{\lambda_{0}, \lambda_{2}, \lambda_{3}\right\}$,

$S_{1}=\left\{\lambda_{1}, \lambda_{3}\right\}$,

which is resonant since it satisfies the same structure than the subspaces ( 2.9).

A resonant subalgebra can be performed

$W_{R}=W_{0} \oplus W_{1}=S_{0} \times V_{0} \oplus S_{1} \times V_{1}$, with $V_{0}$ and $V_{1}$ being the subspaces of the super-Virasoro algebra. Such resonant $S_{\mathcal{M}}^{(4)}$-expansion of the super-Virasoro reproduces a new infinite-dimensional algebra whose generators are related to the super-Virasoro ones through the semigroup elements as

$\mathcal{J}_{m}=\lambda_{0} \ell_{m}, \quad c_{1}=\lambda_{0} c$,

$\ell \mathcal{P}_{m}=\lambda_{2} \ell_{m}, \quad \ell c_{2}=\lambda_{2} c$,

$\ell^{2} \mathcal{Z}_{m}=\lambda_{4} \ell_{m}, \quad \ell^{2} c_{3}=\lambda_{4} c$,

$\ell^{1 / 2} \mathcal{G}_{r}=\lambda_{1} \mathcal{Q}_{r}, \ell^{3 / 2} \mathcal{H}_{r}=\lambda_{3} \mathcal{Q}_{r}$

Then, using the (anti-)commutation relations of the superVirasoro algebra (2.7) and the multiplication law of the semigroup $S_{\mathcal{M}}^{(4)}$ (4.23), one finds that the (anti-)commutators of the expanded superalgebra are given by

$$
\begin{aligned}
{\left[\mathcal{J}_{m}, \mathcal{J}_{n}\right]=} & (m-n) \mathcal{J}_{m+n}+\frac{c_{1}}{12} m\left(m^{2}-1\right) \delta_{m+n, 0}, \\
{\left[\mathcal{J}_{m}, \mathcal{P}_{n}\right]=} & (m-n) \mathcal{P}_{m+n}+\frac{c_{2}}{12} m\left(m^{2}-1\right) \delta_{m+n, 0}, \\
{\left[\mathcal{P}_{m}, \mathcal{P}_{n}\right]=} & (m-n) \mathcal{Z}_{m+n}+\frac{c_{3}}{12} m\left(m^{2}-1\right) \delta_{m+n, 0}, \\
{\left[\mathcal{J}_{m}, \mathcal{Z}_{n}\right]=} & (m-n) \mathcal{Z}_{m+n}+\frac{c_{3}}{12} m\left(m^{2}-1\right) \delta_{m+n, 0}, \\
{\left[\mathcal{P}_{m}, \mathcal{Z}_{n}\right]=} & \frac{1}{\ell^{2}}(m-n) \mathcal{P}_{m+n}+\frac{c_{2}}{12 \ell^{2}}\left(m^{3}-m\right) \delta_{m+n, 0}, \\
{\left[\mathcal{Z}_{m}, \mathcal{Z}_{n}\right]=} & \frac{1}{\ell^{2}}(m-n) \mathcal{Z}_{m+n} \\
& +\frac{c 3}{12 \ell^{2}}\left(m^{3}-m\right) \delta_{m+n, 0}, \\
{\left[\mathcal{J}_{m}, \mathcal{G}_{r}\right]=} & \left(\frac{m}{2}-r\right) \mathcal{G}_{m+r}, \quad\left[\mathcal{P}_{m}, \mathcal{G}_{r}\right]=\left(\frac{m}{2}-r\right) \mathcal{H}_{m+r}, \\
{\left[\mathcal{J}_{m}, \mathcal{H}_{r}\right]=} & \left(\frac{m}{2}-r\right) \mathcal{H}_{m+r}, \quad\left[\mathcal{P}_{m}, \mathcal{H}_{r}\right] \\
= & \frac{1}{\ell^{2}}\left(\frac{m}{2}-r\right) \mathcal{G}_{m+r}, \\
{\left[\mathcal{Z}_{m}, \mathcal{G}_{r}\right]=} & \frac{1}{\ell^{2}}\left(\frac{m}{2}-r\right) \mathcal{G}_{m+r}, \quad\left[\mathcal{Z}_{m}, \mathcal{H}_{r}\right] \\
= & \frac{1}{\ell^{2}}\left(\frac{m}{2}-r\right) \mathcal{H}_{m+r}, \\
\left\{\mathcal{H}_{r}, \mathcal{H}_{s}\right\}= & \frac{1}{\ell^{2}} \mathcal{P}_{r+s}+\frac{c_{2}}{6 \ell^{2}}\left(r^{2}-\frac{1}{4}\right) \delta_{r+s, 0} \\
\left\{\mathcal{G}_{r}, \mathcal{H}_{s}\right\}= & \mathcal{P}_{r+s}+\frac{c_{2}}{6}\left(r^{2}-\frac{1}{4}\right) \delta_{r+s, 0}, \\
\mathcal{Z}_{r+s}+\frac{c_{3}}{6}\left(r^{2}-\frac{1}{4}\right) \delta_{r+s, 0} &
\end{aligned}
$$

Such infinite-dimensional superalgebra is a supersymmetric extension of the enlarged $B M S_{3}$ algebra presented in [34] and turns out to be the infinite-dimensional lift of the minimal AdS-Lorentz superalgebra introduced in [53]. In fact, one can note that the set of generators $\left\{\mathcal{J}_{m}, \mathcal{P}_{m}, Z_{m}, \mathcal{G}_{r}, \mathcal{H}_{r}\right\}$ with $m=0, \pm 1$ and $r= \pm \frac{1}{2}$ defines a finite subalgebra which reproduces the minimal AdS-Lorentz superalgebra spanned by $\left\{J_{a}, P_{a}, Z_{a}, Q, \Sigma\right\}$ through the change of basis (4.6) considered in the deformed super- $\widetilde{B M S} S_{3}$ case. In par- 
ticular, the (anti-)commutators of the minimal AdS-Lorentz superalgebra are given by (B.1) and

$$
\begin{aligned}
{\left[J_{a}, Q_{\alpha}\right] } & =\frac{1}{2}\left(\Gamma_{a}\right)_{\alpha}^{\beta} Q_{\beta}, \quad\left[J_{a}, \Sigma_{\alpha}\right] \\
& =\frac{1}{2}\left(\Gamma_{a}\right)_{\alpha}^{\beta} \Sigma_{\beta}, \\
{\left[P_{a}, Q_{\alpha}\right] } & =\frac{1}{2}\left(\Gamma_{a}\right)_{\alpha}^{\beta} \Sigma_{\beta}, \quad\left[P_{a}, \Sigma_{\alpha}\right] \\
& =\frac{1}{2 \ell^{2}}\left(\Gamma_{a}\right)_{\alpha}^{\beta} Q_{\beta}, \\
{\left[Z_{a}, Q_{\alpha}\right] } & =\frac{1}{2 \ell^{2}}\left(\Gamma_{a}\right)_{\alpha}^{\beta} Q_{\beta}, \quad\left[Z_{a}, \Sigma_{\alpha}\right] \\
& =\frac{1}{2 \ell^{2}}\left(\Gamma_{a}\right)_{\alpha}^{\beta} \Sigma_{\beta}, \\
\left\{Q_{\alpha}, Q_{\beta}\right\} & =\frac{1}{2}\left(C \Gamma^{a}\right)_{\alpha \beta} P_{a},\left\{Q_{\alpha}, \Sigma_{\beta}\right\} \\
& =\frac{1}{2}\left(C \Gamma^{a}\right)_{\alpha \beta} Z_{a}, \\
\left\{\Sigma_{\alpha}, \Sigma_{\beta}\right\} & =\frac{1}{2 \ell^{2}}\left(C \Gamma^{a}\right)_{\alpha \beta} P_{a} .
\end{aligned}
$$

Further supersymmetric extensions of the AdS-Lorentz have also been explored in four spacetime dimensions allowing to introduce a generalized cosmological constant term in supergravity [91-94]. The (anti-)commutation relations (4.29) can alternatively be obtained as an $S$-expansion of the superLorentz algebra using the same semigroup $S_{\mathcal{M}}^{(4)}$. On the other hand, as it bosonic version, the limit $\ell \rightarrow \infty$ of the minimal AdS-Lorentz superalgebra reproduces the minimal Maxwell superalgebra (4.7). It is interesting to notice that such limit can also be applied at the infinite-dimensional level. Indeed the limit $\ell \rightarrow \infty$ applied to the minimal enlarged super$B M S_{3}$ algebra leads to the minimal deformed super- $\widetilde{B M S_{3}}$ algebra obtained previously. The following diagram summarizes the expansion and limit relations:

$$
\begin{array}{rlrl}
Q_{r} & =\frac{1}{2}\left(\ell^{1 / 2} \mathcal{G}_{r}+\ell^{3 / 2} \mathcal{H}_{r}\right), & & \bar{Q}_{r}=\frac{i}{2}\left(\ell^{1 / 2} \mathcal{G}_{r}-\ell^{3 / 2} \mathcal{H}_{r}\right), \\
c^{ \pm}=\frac{1}{2}\left(\ell^{2} c_{3} \pm \ell c_{2}\right), & \hat{c}=\left(c_{1}-\ell^{2} c_{3}\right) .
\end{array}
$$

Specifically, the (anti-)commutators are given by

$$
\begin{aligned}
& {\left[\mathcal{L}_{m}^{+}, \mathcal{L}_{n}^{+}\right]=(m-n) \mathcal{L}_{m+n}^{+}+\frac{c^{+}}{12} m\left(m^{2}-1\right) \delta_{m+n, 0},} \\
& {\left[\mathcal{L}_{m}^{-}, \mathcal{L}_{n}^{-}\right]=(m-n) \mathcal{L}_{m+n}^{-}+\frac{c^{-}}{12} m\left(m^{2}-1\right) \delta_{m+n, 0},} \\
& {\left[\hat{\mathcal{L}}_{m}, \hat{\mathcal{L}}_{n}\right]=(m-n) \hat{\mathcal{L}}_{m+n}+\frac{\hat{c}}{12} m\left(m^{2}-1\right) \delta_{m+n, 0},} \\
& {\left[\mathcal{L}_{m}^{+}, \mathcal{Q}_{r}\right]=\left(\frac{m}{2}-r\right) \mathcal{Q}_{m+r},} \\
& {\left[\mathcal{L}_{m}^{-}, \overline{\mathcal{Q}}_{r}\right]=\left(\frac{m}{2}-r\right) \overline{\mathcal{Q}}_{m+r},} \\
& \left\{\mathcal{Q}_{r}, \mathcal{Q}_{s}\right\}=\mathcal{L}_{r+s}^{+}+\frac{c^{+}}{6}\left(r^{2}-\frac{1}{4}\right) \delta_{r+s, 0}, \\
& \left\{\overline{\mathcal{Q}}_{r}, \overline{\mathcal{Q}}_{s}\right\}=\mathcal{L}_{r+s}^{-}+\frac{c^{-}}{6}\left(r^{2}-\frac{1}{4}\right) \delta_{r+s, 0} .
\end{aligned}
$$

This corresponds to the direct sum vir $\oplus \mathfrak{s v i r} \oplus \mathfrak{s v i r}$ and can be seen as the direct sum of the $(1,1)$ superconformal algebra (3.21) and the Virasoro algebra. Naturally the occurrence of such structure is due to the fact that the finite AdS-Lorentz superalgebra is, in three spacetime dimensions, isomorphic to three copies of the $\mathfrak{s o}(2,1)$ algebra, two of which augmented by supersymmetry.

\subsection{2 $\mathcal{N}=2$ enlarged super- $B M S_{3}$ algebra}

$\mathrm{A} \mathcal{N}=(2,0)$ enlarged super- $B M S_{3}$ algebra can be obtained considering the same semigroup $S_{\mathcal{M}}^{(4)}$ but starting from the $\mathcal{N}=2$ super-Virasoro algebra (4.8). Let us consider the subspace decomposition $\mathfrak{s v i r}_{2}=V_{0} \oplus V_{1}$, with

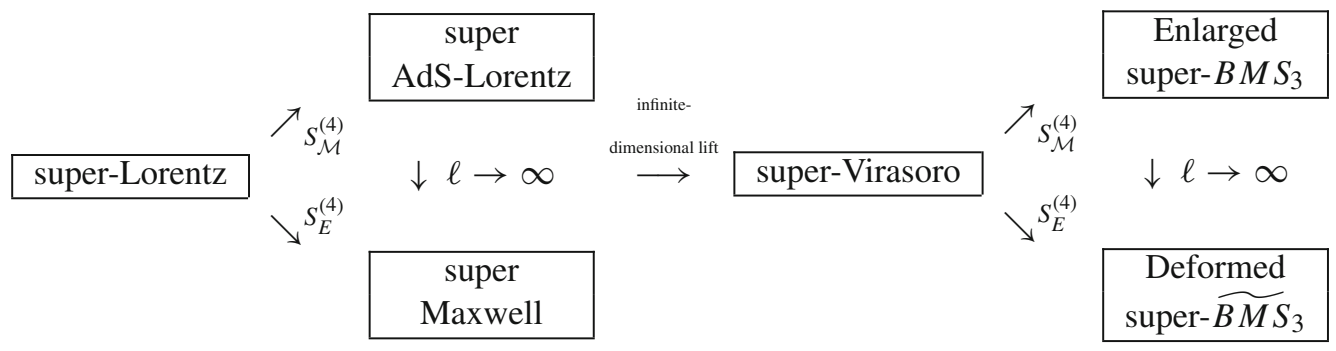

There is an alternative basis in which the enlarged super$B M S_{3}$ algebra can be rewritten. Indeed three copies of the Virasoro algebra, two of which augmented by supersymmetry, are revealed after the following redefinitions:

$$
\begin{aligned}
& \mathcal{L}_{m}^{+}=\frac{1}{2}\left(\ell^{2} \mathcal{Z}_{m}+\ell \mathcal{P}_{m}\right), \quad \mathcal{L}_{m}^{-}=\frac{1}{2}\left(\ell^{2} \mathcal{Z}_{-m}-\ell \mathcal{P}_{-m}\right), \\
& \hat{\mathcal{L}}_{m}=\mathcal{J}_{-m}-\ell^{2} \mathcal{Z}_{-m},
\end{aligned}
$$

$$
\begin{aligned}
& V_{0}=\left\{\ell_{m}, \mathcal{R}_{m}, c\right\}, \\
& V_{1}=\left\{Q_{r}^{i}\right\}
\end{aligned}
$$

which satisfies a graded Lie algebra (2.9). Let $S_{\mathcal{M}}^{(4)}=$ $\left\{\lambda_{0}, \lambda_{1}, \lambda_{2}, \lambda_{3}, \lambda_{4}\right\}$ be the relevant semigroup whose elements satisfy the multiplication law (4.23) and let $S_{\mathcal{M}}^{(4)}=$ $S_{0} \cup S_{1}$ be the resonant subset decomposition with 
$S_{0}=\left\{\lambda_{0}, \lambda_{2}, \lambda_{4}\right\}$,

$S_{1}=\left\{\lambda_{1}, \lambda_{3}\right\}$,

which satisfies the same structure as the subspaces (4.32).

After applying a resonant $S_{\mathcal{M}}^{(4)}$-expansion to the $\mathcal{N}=2$ super-Virasoro algebra one finds a new $\mathcal{N}=2$ infinitedimensional superalgebra spanned by the set $\left\{\mathcal{J}_{m}, \mathcal{P}_{m}, \mathcal{Z}_{m}\right.$, $\left.\mathrm{T}_{m}, \mathrm{~B}_{m}, \mathrm{Z}_{m}, \mathcal{G}_{r}^{i}, \mathcal{H}_{r}^{i}, c_{1}, c_{2}, c_{3}\right\}$ whose generators are related to the super-Virasoro ones through the semigroup elements as follows

$\mathcal{J}_{m}=\lambda_{0} \ell_{m}, \quad c_{1}=\lambda_{0} c$

$\ell \mathcal{P}_{m}=\lambda_{2} \ell_{m}, \quad \ell c_{2}=\lambda_{2} c$,

$\ell^{2} \mathcal{Z}_{m}=\lambda_{4} \ell_{m}, \quad \ell^{2} c_{3}=\lambda_{4} c$,

$\mathrm{T}_{m}=\lambda_{0} \mathcal{R}_{m}, \quad \ell \mathrm{B}_{m}=\lambda_{2} \mathcal{R}_{m}$,

$\ell^{2} \mathrm{z}_{m}=\lambda_{4} \mathcal{R}_{m}$,

$\ell^{1 / 2} \mathcal{G}_{r}=\lambda_{1} \mathcal{Q}_{r}, \ell^{3 / 2} \mathcal{H}_{r}=\lambda_{3} \mathcal{Q}_{r}$.

One can show that the expanded generators satisfy an $\mathcal{N}=$ 2 supersymmetric extension of the enlarged $B M S_{3}$ algebra (B.3) endowed with internal symmetry algebra. In particular, the (anti-)commutation relations can directly be obtained by combining the original (anti-)commutation relations of the $\mathcal{N}=2$ super-Virasoro algebra (4.8) and the multiplication law of the semigroup (4.23). Indeed, the $\mathcal{N}=2$ enlarged super- $B M S_{3}$ algebra is given by its bosonic subalgebra (B.3) and

$$
\begin{aligned}
{\left[\mathcal{J}_{m}, \mathrm{~T}_{n}\right] } & =-n \mathrm{~T}_{m+n}, \quad\left[\mathcal{P}_{m}, \mathrm{~T}_{n}\right]=-n \mathrm{~B}_{m+n}, \\
{\left[\mathcal{Z}_{m}, \mathrm{~T}_{n}\right] } & =-n \mathrm{Z}_{m+n}, \quad\left[\mathcal{J}_{m}, \mathrm{~B}_{n}\right]=-n \mathrm{~B}_{m+n}, \\
{\left[\mathcal{P}_{m}, \mathrm{~B}_{n}\right] } & =-n \mathrm{Z}_{m+n}, \quad\left[\mathcal{Z}_{m}, \mathrm{~B}_{n}\right]=-\frac{1}{\ell^{2}} n \mathrm{~B}_{m+n}, \\
{\left[\mathcal{J}_{m}, \mathrm{Z}_{n}\right] } & =-n \mathrm{Z}_{m+n}, \quad\left[\mathcal{P}_{m}, \mathrm{Z}_{n}\right]=-\frac{1}{\ell^{2}} n \mathrm{~B}_{m+n}, \\
{\left[\mathcal{Z}_{m}, \mathrm{Z}_{n}\right] } & =-\frac{1}{\ell^{2}} n \mathrm{Z}_{m+n}, \\
{\left[\mathrm{~T}_{m}, \mathrm{~T}_{n}\right] } & =\frac{c_{1}}{3} m \delta_{m+n, 0}, \quad\left[\mathrm{~T}_{m}, \mathrm{~B}_{n}\right]=\frac{c_{2}}{3} m \delta_{m+n, 0}, \\
{\left[\mathrm{~T}_{m}, \mathrm{Z}_{n}\right] } & =\frac{c_{3}}{3} m \delta_{m+n, 0}, \quad\left[\mathrm{~B}_{m}, \mathrm{~B}_{n}\right]=\frac{c_{3}}{3} m \delta_{m+n, 0}, \\
{\left[\mathrm{~B}_{m}, \mathrm{Z}_{n}\right] } & =\frac{c_{2}}{3 \ell^{2}} m \delta_{m+n, 0},\left[\mathrm{Z}_{m}, \mathrm{Z}_{n}\right]=\frac{c_{3}}{3 \ell^{2}} m \delta_{m+n, 0}, \\
{\left[\mathcal{J}_{m}, \mathcal{G}_{r}^{i}\right] } & =\left(\frac{m}{2}-r\right) \mathcal{G}_{m+r}^{i}, \quad\left[\mathcal{P}_{m}, \mathcal{G}_{r}^{i}\right] \\
& =\left(\frac{m}{2}-r\right) \mathcal{H}_{m+r}^{i}, \\
{\left[\mathcal{J}_{m}, \mathcal{H}_{r}^{i}\right] } & =\left(\frac{m}{2}-r\right) \mathcal{H}_{m+r}^{i}, \quad\left[\mathcal{P}_{m}, \mathcal{H}_{r}^{i}\right] \\
& =\frac{1}{\ell^{2}}\left(\frac{m}{2}-r\right) \mathcal{G}_{m+r}^{i}, \\
{\left[\mathcal{Z}_{m}, \mathcal{G}_{r}^{i}\right] } & =\frac{1}{\ell^{2}}\left(\frac{m}{2}-r\right) \mathcal{G}_{m+r}^{i}, \quad\left[\mathcal{Z}_{m}, \mathcal{H}_{r}^{i}\right] \\
& =\frac{1}{\ell^{2}}\left(\frac{m}{2}-r\right) \mathcal{H}_{m+r}^{i},
\end{aligned}
$$

$$
\begin{aligned}
{\left[\mathcal{G}_{r}^{i}, \mathrm{~T}_{m}\right]=} & \epsilon^{i j} \mathcal{G}_{m+r}^{j}, \quad\left[\mathcal{G}_{r}^{i}, \mathrm{~B}_{m}\right]=\epsilon^{i j} \mathcal{H}_{m+r}^{j}, \\
{\left[\mathcal{H}_{r}^{i}, \mathrm{~T}_{m}\right]=} & \epsilon^{i j} \mathcal{H}_{m+r}^{j}, \quad\left[\mathcal{G}_{r}^{i}, \mathrm{Z}_{m}\right]=\frac{1}{\ell^{2}} \epsilon^{i j} \mathcal{G}_{m+r}^{j}, \\
{\left[\mathcal{H}_{r}^{i}, \mathrm{~B}_{m}\right]=} & \frac{1}{\ell^{2}} \epsilon^{i j} \mathcal{G}_{m+r}^{j}, \quad\left[\mathcal{H}_{r}^{i}, \mathrm{Z}_{m}\right]=\frac{1}{\ell^{2}} \epsilon^{i j} \mathcal{H}_{m+r}^{j}, \\
\left\{\mathcal{G}_{r}^{i}, \mathcal{G}_{s}^{j}\right\}= & \delta^{i j}\left[\mathcal{P}_{r+s}+\frac{c_{2}}{6}\left(r^{2}-\frac{1}{4}\right) \delta_{r+s, 0}\right] \\
& -2 \epsilon^{i j}(r-s) \mathrm{B}_{r+s}, \\
\left\{\mathcal{G}_{r}^{i}, \mathcal{H}_{s}^{j}\right\}= & \delta^{i j}\left[\mathcal{Z}_{r+s}+\frac{c_{3}}{6}\left(r^{2}-\frac{1}{4}\right) \delta_{r+s, 0}\right] \\
& -2 \epsilon^{i j}(r-s) \mathrm{Z}_{r+s}, \\
\left\{\mathcal{H}_{r}^{i}, \mathcal{H}_{s}^{j}\right\}= & \frac{\delta^{i j}}{\ell^{2}}\left[\mathcal{P}_{r+s}+\frac{c_{2}}{6}\left(r^{2}-\frac{1}{4}\right) \delta_{r+s, 0}\right] \\
& -\frac{2}{\ell^{2}} \epsilon^{i j}(r-s) \mathrm{B}_{r+s} .
\end{aligned}
$$

The $\mathcal{N}=2$ supersymmetric extension of the enlarged $B M S_{3}$ algebra requires the introduction of new bosonic generators $\left\{\mathrm{T}_{m}, \mathrm{~B}_{m}, \mathrm{Z}_{m}\right\}$ which satisfy internal symmetry algebras. Their presence is due to the R-symmetry generator appearing in the $\mathcal{N}=2$ super-Virasoro algebra. Interestingly, a particular redefinition of the generators allows us to rewrite the present infinite-dimensional superalgebra as three copies of the Virasoro algebra, two of which are augmented by supersymmetry, endowed with a $\hat{\mathfrak{u}}(1)$ current algebra. In particular, the structure in presence of $\hat{\mathfrak{u}}(1)$ current generators is apparent by considering the following redefinitions

$$
\begin{aligned}
\mathcal{L}_{m}^{+} & =\frac{1}{2}\left(\ell^{2} \mathcal{Z}_{m}+\ell \mathcal{P}_{m}\right), & & \mathcal{L}_{m}^{-}=\frac{1}{2}\left(\ell^{2} \mathcal{Z}_{-m}-\ell \mathcal{P}_{-m}\right), \\
\hat{\mathcal{L}}_{m} & =\mathcal{J}_{-m}-\ell^{2} \mathcal{Z}_{-m}, & & \\
\mathfrak{k}_{m}^{+} & =\frac{1}{2}\left(\ell \mathrm{B}_{m}+\ell^{2} \mathrm{Z}_{m}\right), & & \mathfrak{k}_{m}^{-}=\frac{1}{2}\left(\ell \mathrm{B}_{m}-\ell^{2} \mathrm{Z}_{m}\right), \\
\hat{\mathfrak{k}}_{m} & =\frac{1}{2}\left(\mathrm{~T}_{m}-\ell^{2} \mathrm{Z}_{m}\right), & & \\
Q_{r}^{i} & =\frac{1}{2}\left(\ell^{1 / 2} \mathcal{G}_{r}^{i}+\ell^{3 / 2} \mathcal{H}_{r}^{i}\right), & & \bar{Q}_{r}^{i}=\frac{i}{2}\left(\ell^{1 / 2} \mathcal{G}_{r}^{i}-\ell \mathcal{H}_{r}^{i}\right), \\
c^{ \pm} & =\frac{1}{2}\left(\ell^{2} c_{3} \pm \ell c_{2}\right), & & \hat{c}=\left(c_{1}-\ell^{2} c_{3}\right) .
\end{aligned}
$$

With these redefinitions the (anti-)commutation relations (B.3), ( 4.35) and (4.36) change into

$$
\begin{aligned}
{\left[\mathcal{L}_{m}^{ \pm}, \mathcal{L}_{n}^{ \pm}\right] } & =(m-n) \mathcal{L}_{m+n}^{ \pm}+\frac{c^{ \pm}}{12} m\left(m^{2}-1\right) \delta_{m+n, 0}, \\
{\left[\hat{\mathcal{L}}_{m}, \hat{\mathcal{L}}_{n}\right] } & =(m-n) \hat{\mathcal{L}}_{m+n}+\frac{\hat{c}}{12} m\left(m^{2}-1\right) \delta_{m+n, 0}, \\
{\left[\mathcal{L}_{m}^{ \pm}, \mathfrak{k}_{n}^{ \pm}\right] } & =-n \mathfrak{k}_{m+n}^{ \pm}, \quad\left[\hat{\mathcal{L}}_{m}, \hat{\mathfrak{k}}_{n}\right]=-n \hat{\mathfrak{k}}_{m+n}, \\
{\left[\mathfrak{k}_{m}^{ \pm}, \mathfrak{k}_{n}^{ \pm}\right] } & =\frac{c^{ \pm}}{3} m \delta_{m+n, 0}, \quad\left[\hat{\mathfrak{k}}_{m}, \hat{\mathfrak{k}}_{n}\right]=\frac{\hat{c}}{3} m \delta_{m+n, 0}, \\
{\left[\mathcal{L}_{m}^{+}, \mathcal{Q}_{r}^{i}\right] } & =\left(\frac{m}{2}-r\right) \mathcal{Q}_{m+r}^{i}, \quad\left[\mathcal{L}_{m}^{-}, \overline{\mathcal{Q}}_{r}^{i}\right] \\
& =\left(\frac{m}{2}-r\right) \overline{\mathcal{Q}}_{m+r}^{i},
\end{aligned}
$$




$$
\begin{aligned}
{\left[\mathcal{Q}_{r}^{i}, \mathfrak{k}_{m}^{+}\right]=} & \epsilon^{i j} \mathcal{Q}_{m+r}^{j}, \quad\left[\overline{\mathcal{Q}}_{r}^{i}, \mathfrak{k}_{m}^{-}\right]=\epsilon^{i j} \overline{\mathcal{Q}}_{m+r}^{j}, \\
\left\{\mathcal{Q}_{r}^{i}, \mathcal{Q}_{s}^{j}\right\}= & \delta^{i j}\left[\mathcal{L}_{r+s}^{+}+\frac{c^{+}}{6}\left(r^{2}-\frac{1}{4}\right) \delta_{r+s, 0}\right] \\
& -2 \epsilon^{i j}(r-s) \mathfrak{k}_{r+s}^{+}, \\
\left\{\overline{\mathcal{Q}}_{r}^{i}, \overline{\mathcal{Q}}_{s}^{j}\right\}= & \delta^{i j}\left[\mathcal{L}_{r+s}^{-}+\frac{c^{-}}{6}\left(r^{2}-\frac{1}{4}\right) \delta_{r+s, 0}\right] \\
& -2 \epsilon^{i j}(r-s) \mathfrak{k}_{r+s}^{-} .
\end{aligned}
$$

The infinite-dimensional superalgebra (4.38) corresponds to the direct sum of the $(2,2)$ superconformal algebra and the Virasoro algebra endowed with a $\hat{\mathfrak{u}}(1)$ current algebra. In particular the $\hat{\mathfrak{u}}(1)$ current generators $\mathfrak{k}_{m}^{+}$and $\mathfrak{k}_{m}^{-}$ are R-symmetry generators each one belonging to a $\mathcal{N}=$ 2 super-Virasoro algebra. Although such structure seems more natural, the $\mathcal{N}=2$ enlarged super- $B M S_{3}$ algebra reproduces the $\mathcal{N}=2$ deformed super- $\widetilde{B M S}$, algebra (4.12)-(4.14) in the limit $\ell \rightarrow \infty$ considering the basis $\left\{\mathcal{J}_{m}, \mathcal{P}_{m}, \mathcal{Z}_{m}, \mathrm{~T}_{m}, \mathrm{~B}_{m}, \mathrm{Z}_{m}, \mathcal{G}_{r}^{i}, \mathcal{H}_{r}^{i}\right\}$. Naturally, the diagram summarizing the expansion and limit relations appearing in the minimal case can also be reproduced in the $\mathcal{N}=2$ case showing that the expansions and flat limit appearing at the infinite-dimensional level are also present in their finite subalgebras.

On the other hand, one can note that the $\mathcal{N}=2$ AdSLorentz superalgebra endowed with $\mathfrak{s o}$ (2) internal symmetry generators [73] is as a finite subalgebra of the $\mathcal{N}=2$ enlarged super- $B M S_{3}$ algebra. Indeed, the subalgebra spanned by the generators $\mathcal{J}_{0}, \mathcal{J}_{ \pm 1}, \mathcal{P}_{0}, \mathcal{P}_{ \pm 1}, \mathcal{Z}_{0}, \mathcal{Z}_{ \pm 1}, \mathrm{~T}_{0}, \mathrm{~B}_{0}, \mathrm{Z}_{0}, \mathcal{G}_{ \pm \frac{1}{2}}$ and $\mathcal{H}_{ \pm \frac{1}{2}}$ are related to the $\mathcal{N}=2$ AdS-Lorentz superalgebra $\left\{J_{a}, P_{a}, Z_{a}, T, B, Z, Q, \Sigma\right\}$ by considering the redefinitions (4.16).

\subsection{3 $\mathcal{N}=4$ enlarged super- $B M S_{3}$ algebra}

For completeness we provide with the $\mathcal{N}=4$ enlarged super$B M S_{3}$ algebra which corresponds to the infinite-dimensional lift of the $\mathcal{N}=4$ AdS-Lorentz superalgebra endowed with internal symmetry algebra. The new infinite-dimensional superalgebra can be obtained by applying an $S$-expansion of the $\mathcal{N}=4$ super-Virasoro algebra (4.17). In particular, considering $S_{\mathcal{M}}^{(4)}$ as the relevant finite semigroup, whose elements satisfy the multiplication law (4.23), and applying a resonant $S_{\mathcal{M}}^{(4)}$-expansion of the $\mathcal{N}=4$ super-Virasoro algebra we find an expanded $\mathcal{N}=4$ infinite-dimensional superalgebra spanned by the generators

$$
\left\{\mathcal{J}_{m}, \mathcal{P}_{m}, \mathcal{Z}_{m}, \mathrm{~T}_{m}^{a}, \mathrm{~B}_{m}^{a}, \mathrm{z}_{m}^{a}, \mathcal{G}_{r}^{i, \pm}, \mathcal{H}_{r}^{i, \pm}, c_{1}, c_{2}, c_{3}\right\}
$$

which are related to the $\mathcal{N}=4$ super-Virasoro ones through (4.19). One can show that, using the multiplication law (4.23) of the $S_{\mathcal{M}}^{(4)}$ semigroup and the original (anti-)commutators (4.17) of the $\mathcal{N}=4$ super-Virasoro algebra, the expanded generators satisfy a $\mathcal{N}=4$ enlarged super- $B M S_{3}$ algebra. In particular the new infinite-dimensional superalgebra contains the enlarged $B M S_{3}$ algebra (B.3) as a bosonic subalgebra. On the other hand, the set of R-symmetry generators $\left\{\mathrm{T}_{m}^{a}, \mathrm{~B}_{m}^{a}, \mathrm{Z}_{m}^{a}\right\}$ with $a=1,2,3$ obeys the following commutators:

$$
\begin{aligned}
& {\left[\mathcal{J}_{m}, \mathrm{~T}_{n}^{a}\right]=-n \mathrm{~T}_{m+n}^{a}, \quad\left[\mathcal{P}_{m}, \mathrm{~T}_{n}^{a}\right]=-n \mathrm{~B}_{m+n}^{a},} \\
& {\left[\mathcal{Z}_{m}, \mathrm{~T}_{n}^{a}\right]=-n \mathrm{Z}_{m+n}^{a}, \quad\left[\mathcal{J}_{m}, \mathrm{~B}_{n}^{a}\right]=-n \mathrm{~B}_{m+n}^{a},} \\
& {\left[\mathcal{P}_{m}, \mathrm{~B}_{n}^{a}\right]=-n \mathrm{Z}_{m+n}^{a}, \quad\left[\mathcal{Z}_{m}, \mathrm{~B}_{n}^{a}\right]=-\frac{1}{\ell^{2}} n \mathrm{~B}_{m+n}^{a},} \\
& {\left[\mathcal{J}_{m}, \mathrm{z}_{n}^{a}\right]=-n \mathrm{Z}_{m+n}^{a}, \quad\left[\mathcal{P}_{m}, \mathrm{z}_{n}^{a}\right]=-\frac{1}{\ell^{2}} n \mathrm{~B}_{m+n}^{a},} \\
& {\left[\mathcal{Z}_{m}, \mathrm{Z}_{n}^{a}\right]=-\frac{1}{\ell^{2}} n \mathrm{Z}_{m+n}^{a},} \\
& {\left[\mathrm{~T}_{m}^{a}, \mathrm{~T}_{n}^{b}\right]=i \epsilon^{a b c} \mathrm{~T}_{m+n}^{c}+\frac{c_{1}}{12} m \delta^{a b} \delta_{m+n, 0},} \\
& {\left[\mathrm{~T}_{m}^{a}, \mathrm{~B}_{n}^{b}\right]=i \epsilon^{a b c} \mathrm{~B}_{m+n}^{c}+\frac{c_{2}}{12} m \delta^{a b} \delta_{m+n, 0},} \\
& {\left[\mathrm{~T}_{m}^{a}, \mathrm{Z}_{n}^{b}\right]=i \epsilon^{a b c} \mathrm{Z}_{m+n}^{c}+\frac{c_{3}}{12} m \delta^{a b} \delta_{m+n, 0},} \\
& {\left[\mathrm{~B}_{m}^{a}, \mathrm{~B}_{n}^{b}\right]=i \epsilon^{a b c} \mathrm{Z}_{m+n}^{c}+\frac{c_{3}}{12} m \delta^{a b} \delta_{m+n, 0},} \\
& {\left[\mathrm{~B}_{m}^{a}, \mathrm{z}_{n}^{b}\right]=\frac{1}{\ell^{2}}\left(i \epsilon^{a b c} \mathrm{~B}_{m+n}^{c}+\frac{c_{2}}{12} m \delta^{a b} \delta_{m+n, 0}\right),} \\
& {\left[\mathrm{z}_{m}^{a}, \mathrm{z}_{n}^{b}\right]=\frac{1}{\ell^{2}}\left(i \epsilon^{a b c} \mathrm{Z}_{m+n}^{c}+\frac{c_{3}}{12} m \delta^{a b} \delta_{m+n, 0}\right) \text {, }} \\
& {\left[\mathrm{T}_{m}^{a}, \mathcal{G}_{r}^{i,+}\right]=-\frac{1}{2}\left(\sigma^{a}\right)_{j}^{i} \mathcal{G}_{m+r}^{j,+},} \\
& {\left[\mathrm{T}_{m}^{a}, \mathcal{G}_{r}^{i,-}\right]=\frac{1}{2}\left(\bar{\sigma}^{a}\right)_{j}^{i} \mathcal{G}_{m+r}^{j,-},} \\
& {\left[\mathrm{B}_{m}^{a}, \mathcal{G}_{r}^{i,+}\right]=-\frac{1}{2}\left(\sigma^{a}\right)_{j}^{i} \mathcal{H}_{m+r}^{j,+},} \\
& {\left[\mathrm{B}_{m}^{a}, \mathcal{G}_{r}^{i,-}\right]=\frac{1}{2}\left(\bar{\sigma}^{a}\right)_{j}^{i} \mathcal{H}_{m+r}^{j,-},} \\
& {\left[\mathrm{T}_{m}^{a}, \mathcal{H}_{r}^{i,+}\right]=-\frac{1}{2}\left(\sigma^{a}\right)_{j}^{i} \mathcal{H}_{m+r}^{j,+},} \\
& {\left[\mathrm{T}_{m}^{a}, \mathcal{H}_{r}^{i,-}\right]=\frac{1}{2}\left(\bar{\sigma}^{a}\right)_{j}^{i} \mathcal{H}_{m+r}^{j,-},} \\
& {\left[\mathrm{B}_{m}^{a}, \mathcal{H}_{r}^{i,+}\right]=-\frac{1}{2 \ell^{2}}\left(\sigma^{a}\right)_{j}^{i} \mathcal{G}_{m+r}^{j,+},} \\
& {\left[\mathrm{B}_{m}^{a}, \mathcal{H}_{r}^{i,-}\right]=\frac{1}{2 \ell^{2}}\left(\bar{\sigma}^{a}\right)_{j}^{i} \mathcal{G}_{m+r}^{j,-},} \\
& {\left[\mathrm{z}_{m}^{a}, \mathcal{G}_{r}^{i,+}\right]=-\frac{1}{2 \ell^{2}}\left(\sigma^{a}\right)_{j}^{i} \mathcal{G}_{m+r}^{j,+},} \\
& {\left[\mathrm{z}_{m}^{a}, \mathcal{G}_{r}^{i,-}\right]=\frac{1}{2 \ell^{2}}\left(\bar{\sigma}^{a}\right)_{j}^{i} \mathcal{G}_{m+r}^{j,-},} \\
& {\left[\mathrm{Z}_{m}^{a}, \mathcal{H}_{r}^{i,+}\right]=-\frac{1}{2 \ell^{2}}\left(\sigma^{a}\right)_{j}^{i} \mathcal{H}_{m+r}^{j,+},} \\
& {\left[\mathrm{z}_{m}^{a}, \mathcal{H}_{r}^{i,-}\right]=\frac{1}{2 \ell^{2}}\left(\bar{\sigma}^{a}\right)_{j}^{i} \mathcal{H}_{m+r}^{j,-},}
\end{aligned}
$$


Here, $\bar{\sigma}_{i j}^{a}=\sigma_{j i}^{a}$ with $\sigma^{a}$ being the Pauli matrices. Furthermore, the fermionic generators $\mathcal{G}_{r}^{i, \pm}$ and $\mathcal{H}_{r}^{i, \pm}$, with $r= \pm \frac{1}{2}$ satisfy the following (anti-)commutation relations:

$$
\begin{aligned}
{\left[\mathcal{J}_{m}, \mathcal{G}_{r}^{i, \pm}\right] } & =\left(\frac{m}{2}-r\right) \mathcal{G}_{m+r}^{i, \pm}, \quad\left[\mathcal{P}_{m},\right. \\
\left.\mathcal{G}_{r}^{i, \pm}\right] & =\left(\frac{m}{2}-r\right) \mathcal{H}_{m+r}^{i, \pm}, \\
{\left[\mathcal{J}_{m}, \mathcal{H}_{r}^{i, \pm}\right] } & =\left(\frac{m}{2}-r\right) \mathcal{H}_{m+r}^{i, \pm}, \\
{\left[\mathcal{Z}_{m}, \mathcal{G}_{r}^{i, \pm}\right] } & =\frac{1}{\ell^{2}}\left(\frac{m}{2}-r\right) \mathcal{G}_{m+r}^{i, \pm},
\end{aligned}
$$

AdS-Lorentz superalgebra leads to the $\mathcal{N}$-extended Maxwell superalgebra in the limit $\ell \rightarrow \infty$ which results to be the finite subalgebra of the $\mathcal{N}$-extended deformed super- $\widetilde{B M S} S_{3}$ algebra. In particular, the internal symmetry generator $\mathrm{Z}_{m}^{a}$ with $m=0, \pm 1$ becomes a central charge after the flat limit.

Note that the semigroup used to obtain the $\mathcal{N}$-extended enlarged super- $B M S_{3}$ algebra from the $\mathcal{N}$-extended superVirasoro algebra is the same used to recovered the $\mathcal{N}$ extended AdS-Lorentz superalgebra from an $\mathcal{N}$-extended super-Lorentz algebra. The following diagram summarizes the limit and expansion relations present in the new infinitedimensional algebras and their finite subalgebra:

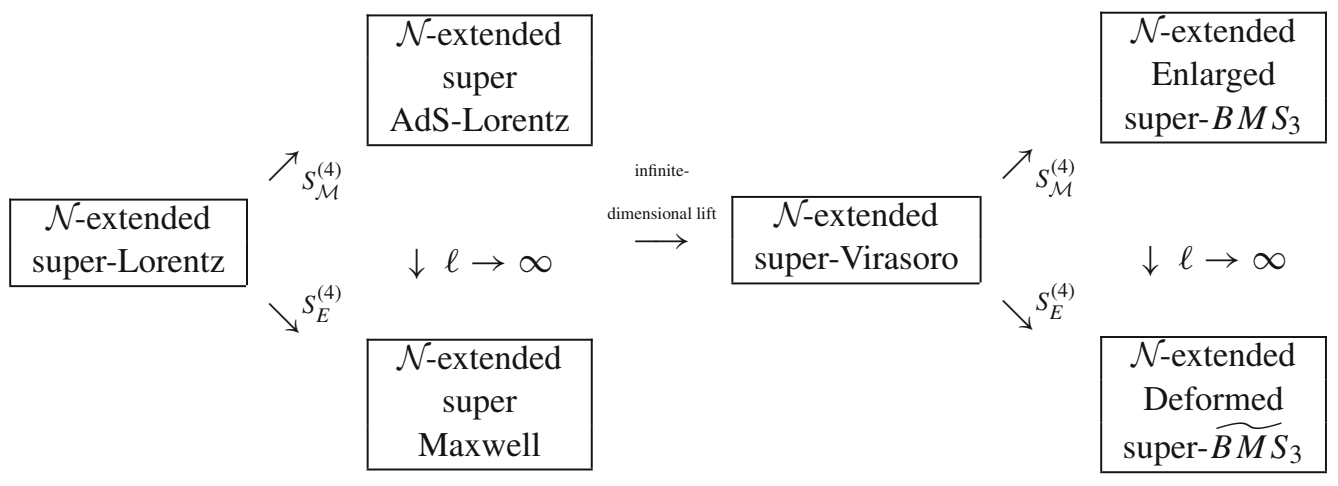

$$
\begin{aligned}
{\left[\mathcal{P}_{m}, \mathcal{H}_{r}^{i, \pm}\right]=} & \frac{1}{\ell^{2}}\left(\frac{m}{2}-r\right) \mathcal{G}_{m+r}^{i, \pm}, \\
{\left[\mathcal{Z}_{m}, \mathcal{H}_{r}^{i, \pm}\right]=} & \frac{1}{\ell^{2}}\left(\frac{m}{2}-r\right) \mathcal{H}_{m+r}^{i, \pm}, \\
\left\{\mathcal{G}_{r}^{i,+}, \mathcal{G}_{s}^{j,-}\right\}= & \delta^{i j}\left[\mathcal{P}_{r+s}+\frac{c_{2}}{6}\left(r^{2}-\frac{1}{4}\right) \delta_{r+s, 0}\right] \\
& -(r-s)\left(\sigma^{a}\right)_{i j} \mathrm{~B}_{r+s}^{a}, \\
\left\{\mathcal{G}_{r}^{i,+}, \mathcal{H}_{s}^{j,-}\right\}= & \delta^{i j}\left[\mathcal{Z}_{r+s}+\frac{c_{3}}{6}\left(r^{2}-\frac{1}{4}\right) \delta_{r+s, 0}\right] \\
& -(r-s)\left(\sigma^{a}\right)_{i j} \mathrm{Z}_{r+s}^{a}, \\
\left\{\mathcal{H}_{r}^{i,+}, \mathcal{H}_{s}^{j,-}\right\}= & \frac{\delta^{i j}}{\ell^{2}}\left[\mathcal{P}_{r+s}+\frac{c_{2}}{6}\left(r^{2}-\frac{1}{4}\right) \delta_{r+s, 0}\right] \\
& -\frac{1}{\ell^{2}}(r-s)\left(\sigma^{a}\right)_{i j} \mathrm{~B}_{r+s}^{a} .
\end{aligned}
$$

It is interesting to note that the $\mathcal{N}=4$ deformed super$\widetilde{B M S}_{3}$ algebra given by (A.3), (4.20) and (4.21) can alternatively be recovered by applying a flat limit $\ell \rightarrow \infty$ to the present $\mathcal{N}=4$ enlarged super- $B M S_{3}$ algebra. In particular, the internal symmetry generator $\mathrm{Z}_{r}^{a}$ is no more a R-symmetry generator after the limit $\ell \rightarrow \infty$. Such flat limit can also be reproduced at the finite subalgebra level. Indeed, the finite set of generators $\left\{\mathcal{J}_{m}, \mathcal{P}_{m}, \mathcal{Z}_{m}, \mathrm{~T}_{m}^{a}, \mathrm{~B}_{m}^{a}, \mathrm{Z}_{m}^{a}, \mathcal{G}_{r}^{i, \pm}, \mathcal{H}_{r}^{i, \pm}\right\}$ with $m=0, \pm 1$ and $r= \pm \frac{1}{2}$ reproduces the $\mathcal{N}=4$ AdS-Lorentz superalgebra [73]. As was discussed in [73], the $\mathcal{N}$-extended

Let us note that the $\mathcal{N}=4$ enlarged super- $B M S_{3}$ can be rewritten in an alternative basis. Indeed a particular redefinition of the generators allows us to rewrite the infinite-dimensional superalgebra as three copies of the Virasoro algebra, two of which augmented by supersymmetry, endowed with $\mathfrak{s u}(2)$ current generators:

$$
\begin{aligned}
{\left[\mathcal{L}_{m}, \mathcal{L}_{n}\right] } & =(m-n) \mathcal{L}_{m+n}+\frac{c}{12} m\left(m^{2}-1\right) \delta_{m+n, 0}, \\
{\left[\overline{\mathcal{L}}_{m}, \overline{\mathcal{L}}_{n}\right] } & =(m-n) \overline{\mathcal{L}}_{m+n}+\frac{\bar{c}}{12} m\left(m^{2}-1\right) \delta_{m+n, 0}, \\
{\left[\hat{\mathcal{L}}_{m}, \hat{\mathcal{L}}_{n}\right] } & =(m-n) \hat{\mathcal{L}}_{m+n}+\frac{\hat{c}}{12} m\left(m^{2}-1\right) \delta_{m+n, 0}, \\
{\left[\mathcal{L}_{m}, \mathfrak{k}_{n}^{a}\right] } & =-n \mathfrak{k}_{m+n}^{a}, \quad\left[\overline{\mathcal{L}}_{m}, \overline{\mathfrak{k}}_{n}^{a}\right]=-n \overline{\mathfrak{k}}_{m+n}^{a}, \\
{\left[\hat{\mathcal{L}}_{m}, \hat{\mathfrak{k}}_{n}^{a}\right] } & =-n \hat{\mathfrak{k}}_{m+n}^{a}, \\
{\left[\mathfrak{k}_{m}^{a}, \mathfrak{k}_{n}^{b}\right] } & =i \epsilon^{a b c} \mathfrak{k}_{m+n}^{c}+\frac{c}{12} m \delta^{a b} \delta_{m+n, 0}, \\
{\left[\overline{\mathfrak{k}}_{m}^{a}, \overline{\mathfrak{k}}_{n}^{b}\right] } & =i \epsilon^{a b c} \overline{\mathfrak{k}}_{m+n}^{c}+\frac{\bar{c}}{12} m \delta^{a b} \delta_{m+n, 0}, \\
{\left[\hat{\mathfrak{k}}_{m}^{a}, \hat{\mathfrak{k}}_{n}^{b}\right] } & =i \epsilon^{a b c} \hat{\mathfrak{k}}_{m+n}^{c}+\frac{\hat{c}}{12} m \delta^{a b} \delta_{m+n, 0}, \\
{\left[\mathcal{L}_{m}, \mathcal{Q}_{r}^{i, \pm}\right] } & =\left(\frac{m}{2}-r\right) \mathcal{Q}_{m+r}^{i, \pm}, \\
{\left[\overline{\mathcal{L}}_{m}, \overline{\mathcal{Q}}_{r}^{i, \pm}\right] } & =\left(\frac{m}{2}-r\right) \overline{\mathcal{Q}}_{m+r}^{i, \pm}, \\
{\left[\mathfrak{k}_{m}^{a}, \mathcal{Q}_{r}^{i,+}\right] } & =-\frac{1}{2}\left(\sigma^{a}\right)_{j}^{i} \mathcal{Q}_{m+r}^{j,+},
\end{aligned}
$$




$$
\begin{aligned}
{\left[\mathfrak{k}_{m}^{a}, \mathcal{Q}_{r}^{i,-}\right]=} & \frac{1}{2}\left(\bar{\sigma}^{a}\right)_{j}^{i} \mathcal{Q}_{m+r}^{j,-}, \\
{\left[\overline{\mathfrak{k}}_{m}^{a}, \overline{\mathcal{Q}}_{r}^{i,+}\right]=} & -\frac{1}{2}\left(\sigma^{a}\right)_{j}^{i} \overline{\mathcal{Q}}_{m+r}^{j,+}, \\
{\left[\overline{\mathfrak{k}}_{m}^{a}, \overline{\mathcal{Q}}_{r}^{i,-}\right]=} & \frac{1}{2}\left(\bar{\sigma}^{a}\right)_{j}^{i} \overline{\mathcal{Q}}_{m+r}^{j,-}, \\
\left\{\mathcal{Q}_{r}^{i,+}, \mathcal{Q}_{s}^{j,-}\right\}= & \delta^{i j}\left[\mathcal{L}_{r+s}+\frac{c}{6}\left(r^{2}-\frac{1}{4}\right) \delta_{r+s, 0}\right] \\
& -(r-s)\left(\sigma^{a}\right)_{i j} \mathfrak{k}_{r+s}^{a}, \\
\left\{\overline{\mathcal{Q}}_{r}^{i,+}, \overline{\mathcal{Q}}_{s}^{j,-}\right\}= & \delta^{i j}\left[\overline{\mathcal{L}}_{r+s}+\frac{\bar{c}}{6}\left(r^{2}-\frac{1}{4}\right) \delta_{r+s, 0}\right] \\
& -(r-s)\left(\sigma^{a}\right)_{i j} \overline{\mathfrak{k}}_{r+s}^{a} .
\end{aligned}
$$

Such structure is revealed by considering the following redefinitions

$$
\begin{aligned}
\mathcal{L}_{m} & =\frac{1}{2}\left(\ell^{2} \mathcal{Z}_{m}+\ell \mathcal{P}_{m}\right), \\
\overline{\mathcal{L}}_{m} & =\frac{1}{2}\left(\ell^{2} \mathcal{Z}_{-m}-\ell \mathcal{P}_{-m}\right), \\
\hat{\mathcal{L}}_{m} & =\mathcal{J}_{-m}-\ell^{2} \mathcal{Z}_{-m}, \\
\mathfrak{k}_{m}^{a} & =\frac{1}{2}\left(\ell \mathrm{B}_{m}^{a}+\ell^{2} \mathrm{Z}_{m}^{a}\right), \\
\overline{\mathfrak{k}}_{m}^{a} & =\frac{1}{2}\left(\ell \mathrm{B}_{m}^{a}-\ell^{2} \mathrm{Z}_{m}^{a}\right), \\
\hat{\mathfrak{k}}_{m}^{a} & =\frac{1}{2}\left(\mathrm{~T}_{m}^{a}-\ell^{2} \mathrm{Z}_{m}^{a}\right), \\
Q_{r}^{i, \pm} & =\frac{1}{2}\left(\ell^{1 / 2} \mathcal{G}_{r}^{i, \pm}+\ell^{3 / 2} \mathcal{H}_{r}^{i, \pm}\right), \\
\bar{Q}_{r}^{i, \pm} & =\frac{i}{2}\left(\ell^{1 / 2} \mathcal{G}_{r}^{i, \pm}-\ell \mathcal{H}_{r}^{i, \pm}\right), \\
c & =\frac{1}{2}\left(\ell^{2} c_{3}+\ell c_{2}\right), \bar{c}=\frac{1}{2}\left(\ell^{2} c_{3}-\ell c_{2}\right), \\
\hat{c} & =\left(c_{1}-\ell^{2} c_{3}\right) .
\end{aligned}
$$

Thus the $\mathcal{N}=4$ enlarged super- $B M S_{3}$ algebra given by (B.3), (4.40), (4.41) and (4.42) can be seen as the direct sum of the $(4,4)$ superconformal algebra and the Virasoro algebra endowed with a $\mathfrak{s u}$ (2) current algebra. In particular, the $\mathfrak{s u}$ (2) current generators $\mathfrak{k}_{m}^{a}$ and $\overline{\mathfrak{k}}_{m}^{a}$ present in the $(4,4)$ superconformal algebra correspond to $\mathfrak{s u}$ (2) R-symmetry generators.

\subsubsection{Non-standard enlarged super-BMS $S_{3}$ algebra}

An alternative supersymmetric extension of the so-called enlarged $\mathrm{BMS}_{3}$ algebra (B.3) can be obtained considering a different semigroup and a different starting infinitedimensional algebra.

Let us consider the superconformal algebra:

$$
\begin{aligned}
{\left[\mathcal{J}_{m}, \mathcal{J}_{n}\right] } & =(m-n) \mathcal{J}_{m+n}+\frac{c_{1}}{12} m\left(m^{2}-1\right) \delta_{m+n, 0}, \\
{\left[\mathcal{J}_{m}, \mathcal{P}_{n}\right] } & =(m-n) \mathcal{P}_{m+n}+\frac{c_{2}}{12} m\left(m^{2}-1\right) \delta_{m+n, 0}, \\
{\left[\mathcal{P}_{m}, \mathcal{P}_{n}\right] } & =(m-n) \mathcal{J}_{m+n}+\frac{c_{1}}{12} m\left(m^{2}-1\right) \delta_{m+n, 0}, \\
{\left[\mathcal{J}_{m}, \mathcal{G}_{r}\right] } & =\left(\frac{m}{2}-r\right) \mathcal{G}_{m+r}, \\
{\left[\mathcal{P}_{m}, \mathcal{G}_{r}\right] } & =\left(\frac{m}{2}-r\right) \mathcal{G}_{m+r}, \\
\left\{\mathcal{G}_{r}, \mathcal{G}_{s}\right\} & =\mathcal{J}_{r+s}+\mathcal{P}_{r+s}+\frac{\left(c_{1}+c_{2}\right)}{6}\left(r^{2}-\frac{1}{4}\right) \delta_{r+s, 0} .
\end{aligned}
$$

which, as was discussed in Sect. 3.2, can be written as two copies of the Virasoro algebra, one of which is augmented by supersymmetry.

Let $\mathfrak{g}=V_{0} \oplus V_{1} \oplus V_{2}$ be a subspace decomposition where $V_{0}$ corresponds to the Virasoro subalgebra which is generated by $\left\{\mathcal{J}_{m}, c_{1}\right\}, V_{1}$ is the fermionic subspace spanned by $\mathcal{G}_{r}$ and $V_{2}$ is the bosonic subspace generated by the set $\left\{\mathcal{P}_{m}, c_{2}\right\}$. One can notice that the subspaces satisfy

$$
\begin{array}{lll}
{\left[V_{0}, V_{0}\right] \subset V_{0},} & {\left[V_{0}, V_{1}\right] \subset V_{1},} & {\left[V_{0}, V_{2}\right] \subset V_{2},} \\
{\left[V_{1}, V_{2}\right] \subset V_{1},} & {\left[V_{2}, V_{2}\right] \subset V_{0},} & {\left[V_{1}, V_{1}\right] \subset V_{0} \oplus V_{2} .}
\end{array}
$$

Let $S_{\mathcal{M}}^{(2)}=\left\{\lambda_{0}, \lambda_{1}, \lambda_{2}\right\}$ be the relevant semigroup whose elements satisfy

$$
\begin{array}{l|lll}
\lambda_{2} & \lambda_{2} & \lambda_{1} & \lambda_{2} \\
\lambda_{1} & \lambda_{1} & \lambda_{2} & \lambda_{1} \\
\lambda_{0} & \lambda_{0} & \lambda_{1} & \lambda_{2} \\
\hline & \lambda_{0} & \lambda_{1} & \lambda_{2}
\end{array}
$$

and let us consider the following subset decomposition $S_{\mathcal{M}}^{(2)}=S_{0} \cup S_{1} \cup S_{2}$ with

$S_{0}=\left\{\lambda_{0}, \lambda_{2}\right\}$,

$S_{1}=\left\{\lambda_{1}\right\}$,

$S_{2}=\left\{\lambda_{2}\right\}$,

which is resonant since they satisfy the same structure than the subspaces $V_{0}, V_{1}$ and $V_{2}$. Then, the resonant subalgebra is given by

$W_{R}=W_{0} \oplus W_{1} \oplus W_{2}=S_{0} \times V_{0} \oplus S_{1} \times V_{1} \oplus S_{2} \times V_{2}$.

A new supersymmetric extension of the enlarged $B M S_{3}$ algebra is obtained after performing a resonant $S_{\mathcal{M}}^{(2)}$-expansion whose generators are related to the superconformal ones through 
$\tilde{\mathcal{J}}_{m}=\lambda_{0} \mathcal{J}_{m}, \tilde{c}_{1}=\lambda_{0} c_{1}$,

$\tilde{\mathcal{Z}}_{m}=\lambda_{2} \mathcal{J}_{m}, \tilde{c}_{3}=\lambda_{2} c_{1}$,

$\tilde{\mathcal{P}}_{m}=\lambda_{2} \mathcal{P}_{m}, \tilde{c}_{2}=\lambda_{2} c_{2}$,

$\tilde{\mathcal{G}}_{r}=\lambda_{1} \mathcal{G}_{r}$.

Then, using the (anti-)commutation relations of the superconformal algebra (4.47) and the multiplication law of the semigroup (4.49), one find the non-standard enlarged super$\mathrm{BMS}_{3}$ algebra:

$$
\begin{aligned}
& {\left[\tilde{\mathcal{J}}_{m}, \tilde{\mathcal{J}}_{n}\right]=(m-n) \tilde{\mathcal{J}}_{m+n}+\frac{\tilde{c}_{1}}{12} m\left(m^{2}-1\right) \delta_{m+n, 0},} \\
& {\left[\tilde{\mathcal{J}}_{m}, \tilde{\mathcal{P}}_{n}\right]=(m-n) \tilde{\mathcal{P}}_{m+n}+\frac{\tilde{c}_{2}}{12} m\left(m^{2}-1\right) \delta_{m+n, 0},} \\
& {\left[\tilde{\mathcal{P}}_{m}, \tilde{\mathcal{P}}_{n}\right]=(m-n) \tilde{\mathcal{Z}}_{m+n}+\frac{\tilde{c}_{3}}{12} m\left(m^{2}-1\right) \delta_{m+n, 0},} \\
& {\left[\tilde{\mathcal{J}}_{m}, \tilde{\mathcal{Z}}_{n}\right]=(m-n) \tilde{\mathcal{Z}}_{m+n}+\frac{\tilde{c}_{3}}{12} m\left(m^{2}-1\right) \delta_{m+n, 0},} \\
& {\left[\tilde{\mathcal{P}}_{m}, \tilde{\mathcal{Z}}_{n}\right]=(m-n) \tilde{\mathcal{P}}_{m+n}+\frac{\tilde{c}_{2}}{12} m\left(m^{2}-1\right) \delta_{m+n, 0},} \\
& {\left[\tilde{\mathcal{Z}}_{m}, \tilde{\mathcal{Z}}_{n}\right]=(m-n) \tilde{\mathcal{Z}}_{m+n}+\frac{\tilde{c}_{3}}{12} m\left(m^{2}-1\right) \delta_{m+n, 0},} \\
& {\left[\tilde{\mathcal{J}}_{m}, \tilde{\mathcal{G}}_{r}\right]=\left(\frac{m}{2}-r\right) \tilde{\mathcal{G}}_{m+r},} \\
& {\left[\tilde{\mathcal{P}}_{m}, \tilde{\mathcal{G}}_{r}\right]=\left(\frac{m}{2}-r\right) \tilde{\mathcal{G}}_{m+r},} \\
& {\left[\tilde{\mathcal{Z}}_{m}, \tilde{\mathcal{G}}_{r}\right]=\left(\frac{m}{2}-r\right) \tilde{\mathcal{G}}_{m+r},} \\
& \left\{\tilde{\mathcal{G}}_{r}, \tilde{\mathcal{G}}_{s}\right\}=\tilde{\mathcal{Z}}_{r+s}+\tilde{\mathcal{P}}_{r+s} \\
& \quad+\frac{\left(\tilde{c}_{3}+\tilde{c}_{2}\right)}{6}\left(r^{2}-\frac{1}{4}\right) \delta_{r+s, 0} .
\end{aligned}
$$

The new superalgebra obtained corresponds to the infinitedimensional lift of a particular AdS-Lorentz superalgebra introduced in [95]. One can see that the finite subalgebra spanned by $\tilde{\mathcal{J}}_{0}, \tilde{\mathcal{J}}_{1}, \tilde{\mathcal{J}}_{-1}, \tilde{\mathcal{P}}_{0}, \tilde{\mathcal{P}}_{1}, \tilde{\mathcal{P}}_{-1}, \tilde{\mathcal{Z}}_{0}, \tilde{\mathcal{Z}}_{1}, \tilde{\mathcal{Z}}_{-1}, \tilde{\mathcal{G}}_{\frac{1}{2}}$ and $\tilde{\mathcal{G}}_{-\frac{1}{2}}$ are related to the super AdS-Lorentz ones through

$\tilde{\mathcal{J}}_{-1}=-\sqrt{2} \tilde{J}_{0}, \quad \tilde{\mathcal{J}}_{1}=\sqrt{2} \tilde{J}_{1}, \quad \tilde{\mathcal{J}}_{0}=\tilde{J}_{2}$,

$\tilde{\mathcal{P}}_{-1}=-\sqrt{2} \tilde{P}_{0}, \tilde{\mathcal{P}}_{1}=\sqrt{2} \tilde{P}_{1}, \quad \tilde{\mathcal{P}}_{0}=\tilde{P}_{2}$,

$\tilde{\mathcal{Z}}_{-1}=-\sqrt{2} \tilde{Z}_{0}, \tilde{\mathcal{Z}}_{1}=\sqrt{2} \tilde{Z}_{1}, \quad \tilde{\mathcal{Z}}_{0}=\tilde{Z}_{2}$

$\tilde{\mathcal{G}}_{-\frac{1}{2}}=\sqrt{2} \tilde{Q}_{+}, \quad \tilde{\mathcal{G}}_{\frac{1}{2}}=\sqrt{2} \tilde{Q}_{-}$.

As the minimal enlarged super- $B M S_{3}$ algebra (4.27)-(4.28), the non-standard one obtained here can be rewritten as three copies of the Virasoro algebra but only one augmented by supersymmetry,

$$
\begin{aligned}
& {\left[\mathcal{L}_{m}^{+}, \mathcal{L}_{n}^{+}\right]=(m-n) \mathcal{L}_{m+n}^{+}+\frac{c^{+}}{12} m\left(m^{2}-1\right) \delta_{m+n, 0},} \\
& {\left[\mathcal{L}_{m}^{-}, \mathcal{L}_{n}^{-}\right]=(m-n) \mathcal{L}_{m+n}^{-}+\frac{c^{-}}{12} m\left(m^{2}-1\right) \delta_{m+n, 0},} \\
& {\left[\hat{\mathcal{L}}_{m}, \hat{\mathcal{L}}_{n}\right]=(m-n) \hat{\mathcal{L}}_{m+n}+\frac{\hat{c}}{12} m\left(m^{2}-1\right) \delta_{m+n, 0},}
\end{aligned}
$$

$$
\begin{aligned}
& {\left[\mathcal{L}_{m}^{+}, \mathcal{Q}_{r}\right]=\left(\frac{m}{2}-r\right) \mathcal{Q}_{m+r}} \\
& \left\{\mathcal{Q}_{r}, \mathcal{Q}_{s}\right\}=\mathcal{L}_{r+s}^{+}+\frac{c^{+}}{6}\left(r^{2}-\frac{1}{4}\right) \delta_{r+s, 0}
\end{aligned}
$$

This corresponds to the direct sum of the $(1,0)$ superconformal algebra and the Virasoro algebra. Such structure appears after the following redefinitions:

$$
\begin{array}{ll}
\mathcal{L}_{m}^{+}=\frac{1}{2}\left(\tilde{\mathcal{Z}}_{m}+\tilde{\mathcal{P}}_{m}\right), \mathcal{L}_{m}^{-}=\frac{1}{2}\left(\tilde{\mathcal{Z}}_{-m}-\tilde{\mathcal{P}}_{-m}\right), \\
\hat{\mathcal{L}}_{m}=\tilde{\mathcal{J}}_{-m}-\tilde{\mathcal{Z}}_{-m}, \quad Q_{r}=\frac{1}{\sqrt{2}} \tilde{\mathcal{G}}_{r}, \\
c^{ \pm}=\frac{1}{2}\left(\tilde{c}_{3} \pm \tilde{c}_{2}\right), \quad \hat{c}=\left(\tilde{c}_{1}-\tilde{c}_{3}\right) .
\end{array}
$$

The main difference with the enlarged super- $B M S_{3}$ algebra (4.27)-(4.28) introduced previously is the absence of a second spinor charge. Then, it seems that the connection with the deformed super- $\widetilde{B M S} S_{3}$ algebra, which possesses two spinor charges $\mathcal{G}_{r}$ and $\mathcal{H}_{r}$, cannot be done from this non-standard enlarged super- $B M S_{3}$ algebra. Nevertheless, a non-standard deformed super- $\widetilde{B M S_{3}}$ algebra can be obtained considering an Inönü-Wigner contraction to (4.53). Indeed, the rescaling of the generators of ( 4.53)

$$
\begin{aligned}
\tilde{\mathcal{J}}_{m} & \rightarrow \tilde{\mathcal{J}}_{m}, \quad \tilde{\mathcal{P}}_{m} \rightarrow \sigma \tilde{\mathcal{P}}_{m}, \quad \tilde{\mathcal{Z}}_{m} \rightarrow \sigma^{2} \tilde{\mathcal{Z}}_{m}, \quad \tilde{\mathcal{G}}_{r} \rightarrow \sigma \tilde{\mathcal{G}}_{r}, \\
\tilde{c}_{1} & \rightarrow \tilde{c}_{1}, \quad \tilde{c}_{2} \rightarrow \sigma \tilde{c}_{2}, \quad \tilde{c}_{3} \rightarrow \sigma^{2} \tilde{c}_{3},
\end{aligned}
$$

leads to a non-standard deformed super- $\widetilde{B M S}$ algebra in the limit $\sigma \rightarrow \infty$ :

$$
\begin{aligned}
& {\left[\tilde{\mathcal{J}}_{m}, \tilde{\mathcal{J}}_{n}\right]=(m-n) \tilde{\mathcal{J}}_{m+n}+\frac{\tilde{c}_{1}}{12} m\left(m^{2}-1\right) \delta_{m+n, 0},} \\
& {\left[\tilde{\mathcal{J}}_{m}, \tilde{\mathcal{P}}_{n}\right]=(m-n) \tilde{\mathcal{P}}_{m+n}+\frac{\tilde{c}_{2}}{12} m\left(m^{2}-1\right) \delta_{m+n, 0},} \\
& {\left[\tilde{\mathcal{P}}_{m}, \tilde{\mathcal{P}}_{n}\right]=(m-n) \tilde{\mathcal{Z}}_{m+n}+\frac{\tilde{c}_{3}}{12} m\left(m^{2}-1\right) \delta_{m+n, 0},} \\
& {\left[\tilde{\mathcal{J}}_{m}, \tilde{\mathcal{Z}}_{n}\right]=(m-n) \tilde{\mathcal{Z}}_{m+n}+\frac{\tilde{c}_{3}}{12} m\left(m^{2}-1\right) \delta_{m+n, 0},} \\
& {\left[\tilde{\mathcal{J}}_{m}, \tilde{\mathcal{G}}_{r}\right]=\left(\frac{m}{2}-r\right) \tilde{\mathcal{G}}_{m+r},} \\
& \left\{\tilde{\mathcal{G}}_{r}, \tilde{\mathcal{G}}_{s}\right\}=\tilde{\mathcal{Z}}_{r+s}+\frac{\tilde{c}_{3}}{6}\left(r^{2}-\frac{1}{4}\right) \delta_{r+s, 0} .
\end{aligned}
$$

The name "non-standard" is due to its finite subalgebra which corresponds to the so-called non-standard Maxwell superalgebra $[76,96]$. Such supersymmetric extension of the Maxwell algebra has the particularity that the fourmomentum generators $\tilde{P}_{a}$ are no more expressed as bilinear expressions of the fermionic ones leading to an exotic three-dimensional supersymmetric action. As was discussed in [53], a well-defined supergravity action invariant under a supersymmetric extension of the Maxwell algebra requires the introduction of a second spinorial charge. 


\section{Conclusions}

The $S$-expansion procedure has been useful to obtain new (super)symmetries and novel (super)gravity theories. Here, based on the recent applications of the $S$-expansion method in the asymptotic symmetries context [50,51], we have obtained known and new supersymmetric extensions of asymptotic symmetries of diverse three-dimensional gravity theories. By expanding the super-Virasoro algebra we have found the minimal supersymmetric extensions of the asymptotic algebras of the Maxwell gravity [17] and $\mathfrak{s o}(2,2) \oplus \mathfrak{s o}(2,1)$ gravity [34] theories defined in three spacetime dimensions. The new infinite-dimensional superalgebras obtained can be seen as enlargement, extension and deformation of the super- $B M S_{3}$ algebra and corresponds to infinite-dimensional lift of the AdS-Lorentz and Maxwell superalgebras.

The $\mathcal{N}$-extensions of our results have also been considered with $\mathcal{N}=2$ and $\mathcal{N}=4$. We have found that the new $\mathcal{N}$-extended infinite-dimensional superalgebras involve new features. Indeed, in the $\mathcal{N}=2$ case we have shown that $\hat{\mathfrak{u}}$ (1) R-symmetry generators are required. On the other hand, in the $\mathcal{N}=4$ case, we have $\mathfrak{s u}$ (2) R-symmetry generators. Interestingly, we have shown that the $\mathcal{N}$-extended enlarged super- $B M S_{3}$ and the $\mathcal{N}$-extended deformed super$\widetilde{B M S_{3}}$ algebras are related through a flat limit $\ell \rightarrow \infty$. Such limit is not a particularity of the infinite-dimensional superalgebras obtained but is already present at the finite level [53,73].

On the other hand, it is important to clarify that the election of the semigroups to obtain the diverse infinite-dimensional superalgebras is not arbitrary. Indeed, following the results obtained in the bosonic case in [50] and subsequently at the supersymmetric level [51], the semigroups chosen are those used at the finite-dimensional level. ories but also provides us with their asymptotic symmetry. At the bosonic level, we have recently shown that the infinite-dimensional lifts of the Maxwell and AdS-Lorentz algebra obtained as $S$-expansion [50] result to be the respective asymptotic symmetries of the CS gravity theory based on the Maxwell [17] and AdS-Lorentz gravity theories [34], respectively. More recently in [51], we have shown that the semigroup allowing to obtain $\mathcal{N}$-extended Poincaré superalgebras can also be used to obtain $\mathcal{N}$-extended super- $B M S_{3}$ algebras. It is then natural to expect that the novel infinitedimensional superalgebras obtained here are the respective asymptotic supersymmetries of the Maxwell and AdSLorentz supergravity theories. It would be interesting to explore the explicit obtention of our infinite-dimensional superalgebras by imposing suitable boundary conditions. A direct asymptotic symmetry analysis for the Maxwell and $\mathfrak{s o}(2,2) \oplus \mathfrak{s o}(2,1)$ supergravity theory would be approached in a future work.

Another aspect that it would be worth exploring is the connection of our new infinite-dimensional superalgebras with the two-dimensional super Galilean conformal algebra (GCA) [70,71,97]. In particular, one could expect to obtain a deformed super-GCA algebra by contracting the relativistic $(1,1)$ Superconformal $\oplus$ Virasoro algebra obtained here. It would be interesting to evaluate if the deformed super$\widetilde{B M S}_{3}$ algebra introduced here is isomorphic to a deformed super-GCA [work in progress].

As an ending remark: It would be worth it to generalize the recent results obtained with the algebraic expansion method at the non-relativistic level [42,98-101]. It would be interesting to study the non-relativistic version of the Maxwell and AdS-Lorentz superalgebra using the $S$-expansion method.

$$
\begin{aligned}
& \begin{array}{|l|l|l|l|}
\hline \text { Original superalgebra } & S \text { expansion } & \text { Expanded superalgebra } \\
& &
\end{array} \\
& \downarrow \text { infinite-dimensional lift } \\
& \downarrow \text { infinite-dimensional lift }
\end{aligned}
$$

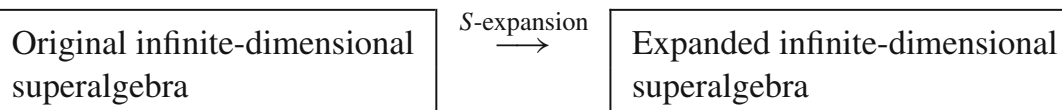

The new results obtained here could have important consequences in further studies of the Maxwell and AdSLorentz supergravity theories. In particular, we conjecture that the new infinite-dimensional structures introduced here are the respective asymptotic supersymmetries of the threedimensional Maxwell and AdS-Lorentz supergravities presented in $[53,73]$. If our conjecture is true, it would mean that the $S$-expansion method not only allows us to construct new and consistent (super)algebras and (super)gravity the-
Acknowledgements This work was supported by the CONICYT-PAI Grant No. 77190078 (P.C.) and FONDECYT Projects No. 11191175 (O.F.) and No. 3170438 (E.R.) . This work was supported by the Research project Code DIN 11/2012 (R.C.) and DINREG 19/2018 (O.F.) of the Universidad Católica de la Santisima Concepción, Chile. R.C., P.C. and O.F. would like to thank to the Dirección de Investigación and Vice-rectoría de Investigación of the Universidad Católica de la Santísima Concepción, Chile, for their constant support. 
Data Availability Statement This manuscript has no associated data or the data will not be deposited. [Authors' comment: The results obtained and presented in the manuscript did not require any data.]

Open Access This article is licensed under a Creative Commons Attribution 4.0 International License, which permits use, sharing, adaptation, distribution and reproduction in any medium or format, as long as you give appropriate credit to the original author(s) and the source, provide a link to the Creative Commons licence, and indicate if changes were made. The images or other third party material in this article are included in the article's Creative Commons licence, unless indicated otherwise in a credit line to the material. If material is not included in the article's Creative Commons licence and your intended use is not permitted by statutory regulation or exceeds the permitted use, you will need to obtain permission directly from the copyright holder. To view a copy of this licence, visit http://creativecomm ons.org/licenses/by/4.0/.

Funded by SCOAP ${ }^{3}$.

\section{A Appendix}

In three spacetime dimensions, the Maxwell algebra has the following non-vanishing commutation relations:

$$
\begin{aligned}
{\left[J_{a}, J_{b}\right] } & =\epsilon_{a b c} J^{c}, \quad\left[J_{a}, P_{b}\right]=\epsilon_{a b c} P^{c}, \\
{\left[J_{a}, Z_{b}\right] } & =\epsilon_{a b c} Z^{c},
\end{aligned}
$$

where $J_{a}$ is the Lorentz generator, $P_{a}$ is the translation generator and $Z_{a}$ is the so-called gravitational Maxwell generator. Such symmetry has been introduced in [18-20] in order to describe a particle moving in a four-dimensional background in presence of a constant electromagnetic field.

As shown in [17,27,28,30], a three-dimensional CS action invariant under the Maxwell symmetry reads

$$
\begin{aligned}
I= & \frac{k}{4 \pi} \int\left[\alpha_{0}\left(\omega^{a} d \omega_{a}+\frac{1}{3} \epsilon_{a b c} \omega^{a} \omega^{b} \omega^{c}\right)+2 \alpha_{1} e^{a} R_{a}\right. \\
& \left.+\alpha_{2}\left(2 R^{a} \sigma_{a}+e^{a} T_{a}\right)-d\left(\alpha_{1} \omega^{e} e_{a}+\alpha_{2} \omega^{a} \sigma_{a}\right)\right],
\end{aligned}
$$

where $e^{a}$ is the vielbein, $\omega^{a}$ corresponds to the spin connection, $\sigma^{a}$ is the gravitational Maxwell gauge field, $R^{a}=$ $d \omega^{a}+\frac{1}{2} \epsilon^{a b c} \omega_{b} \omega_{c}$ is the Lorentz curvature and $T^{a}=D_{\omega} e^{a}$ is the torsion two-form. As was discussed in [17], the vacuum angular momentum and vacuum energy of the stationary configuration are influenced by the gravitational Maxwell field. More recent results have been presented in [102,103] in the dual version of the Maxwell algebra known as Hietarinta algebra [104]. The Hietarinta symmetry appears by interchanging the role of the Maxwell gravitational generator $Z_{a}$ with the momentum generator $P_{a}$.

Interestingly, the boundary dynamics results to be described by an extension and deformation of the $B M S_{3}$ algebra with three independent central charges [17]. Such analysis was based on the charge algebra of the theory in the BMS gauge which includes the solutions of standard asymptotically flat case.
As the Maxwell algebra, the asymptotic symmetry contains an additional Abelian generator $\mathcal{Z}_{m}$ which modifies the $B M S_{3}$ symmetry as follows

$$
\begin{aligned}
& {\left[\mathcal{J}_{m}, \mathcal{J}_{n}\right]=(m-n) \mathcal{J}_{m+n}+\frac{c_{1}}{12}\left(m^{3}-m\right) \delta_{m+n, 0},} \\
& {\left[\mathcal{J}_{m}, \mathcal{P}_{n}\right]=(m-n) \mathcal{P}_{m+n}+\frac{c_{2}}{12}\left(m^{3}-m\right) \delta_{m+n, 0},} \\
& {\left[\mathcal{P}_{m}, \mathcal{P}_{n}\right]=(m-n) \mathcal{Z}_{m+n}+\frac{c_{3}}{12}\left(m^{3}-m\right) \delta_{m+n, 0},} \\
& {\left[\mathcal{J}_{m}, \mathcal{Z}_{n}\right]=(m-n) \mathcal{Z}_{m+n}+\frac{c_{3}}{12}\left(m^{3}-m\right) \delta_{m+n, 0},} \\
& {\left[\mathcal{P}_{m}, \mathcal{Z}_{n}\right]=0} \\
& {\left[\mathcal{Z}_{m}, \mathcal{Z}_{n}\right]=0}
\end{aligned}
$$

Here, the central charges $c_{1}, c_{2}$ and $c_{3}$ are related to the three terms of the CS action (A.2) with

$c_{1}=12 k \alpha_{0}, \quad c_{2}=12 k \alpha_{1}, \quad c_{3}=12 k \alpha_{2}$.

Let us note that the Maxwell algebra (A.1) is a finite subalgebra of the deformed $\widetilde{B M S}_{3}$ algebra (A.3) spanned by $\mathcal{J}_{0}$, $\mathcal{J}_{1}, \mathcal{J}_{-1}, \mathcal{P}_{0}, \mathcal{P}_{1}, \mathcal{P}_{-1}$ and $\mathcal{Z}_{0}, \mathcal{Z}_{1}, \mathcal{Z}_{-1}$ with

$$
\begin{aligned}
& \mathcal{J}_{-1}=-\sqrt{2} J_{0}, \mathcal{J}_{1}=\sqrt{2} J_{1}, \mathcal{J}_{0}=J_{2}, \\
& \mathcal{P}_{-1}=-\sqrt{2} P_{0}, \mathcal{P}_{1}=\sqrt{2} P_{1}, \mathcal{P}_{0}=P_{2}, \\
& \mathcal{Z}_{-1}=-\sqrt{2} Z_{0}, \mathcal{Z}_{1}=\sqrt{2} Z_{1}, \mathcal{Z}_{0}=Z_{2} .
\end{aligned}
$$

\section{B Appendix}

A semi-simple enlargement of the Poincaré symmetry has been introduced in [35-38] which can be seen as the direct sum of the Lorentz and AdS algebra. In three spacetime dimensions, the so-called AdS-Lorentz algebra can be written in the basis $\left\{J_{a}, P_{a}, Z_{a}\right\}$ whose generators satisfy

$$
\begin{aligned}
& {\left[J_{a}, J_{b}\right]=\epsilon_{a b c} J^{c}, \quad\left[P_{a}, P_{b}\right]=\epsilon_{a b c} Z^{c},} \\
& {\left[J_{a}, Z_{b}\right]=\epsilon_{a b c} Z^{c}, \quad\left[Z_{a}, Z_{b}\right]=\frac{1}{\ell^{2}} \epsilon_{a b c} Z^{c},} \\
& {\left[J_{a}, P_{b}\right]=\epsilon_{a b c} P^{c}, \quad\left[Z_{a}, P_{b}\right]=\frac{1}{\ell^{2}} \epsilon_{a b c} P^{c},}
\end{aligned}
$$

where $Z_{a}$ are non-Abelian generators. Interestingly, in such basis, the Maxwell algebra (A.1) can also be obtained as a flat limit $\ell \rightarrow \infty$ of the AdS-Lorentz algebra. Such limit can be reproduced not only at the bosonic level but also at the supersymmetric [53,73,105], non-relativistic [42,101], higher-spin [106] and asymptotic level [34].

The three-dimensional CS gravity action invariant under the AdS-Lorentz algebra (B.1) reads [28,34,37,42,107] 


$$
\begin{aligned}
I_{R}= & \int\left[\alpha_{0}\left(\omega^{a} d \omega_{a}+\frac{1}{3} \epsilon^{a b c} \omega_{a} \omega_{b} \omega_{c}\right)\right. \\
& +\alpha_{1}\left(2 e_{a} R^{a}+\frac{2}{\ell^{2}} e_{a} F^{a}+\frac{1}{3 \ell^{2}} \epsilon^{a b c} e_{a} e_{b} e_{c}\right) \\
& +\alpha_{2}\left(T^{a} e_{a}+\frac{1}{\ell^{2}} \epsilon^{a b c} e_{a} \sigma_{b} e_{c}\right. \\
& \left.\left.+2 \sigma_{a} R^{a}+\frac{2}{\ell^{2}} \sigma_{a} D_{\omega} \sigma^{a}+\frac{1}{3 \ell^{4}} \epsilon^{a b c} \sigma_{a} \sigma_{b} \sigma_{c}\right)\right],
\end{aligned}
$$

where, $R^{a}=d \omega^{a}+\frac{1}{2} \epsilon^{a b c} \omega_{b} \omega_{c}$ is the Lorentz curvature two-form, $T^{a}=D_{\omega} e^{a}$ is the torsion two-form and $F^{a}=$ $D_{\omega} \sigma^{a}+\frac{1}{2 \ell^{2}} \epsilon^{a b c} \sigma_{b} \sigma_{c}$ is the curvature two-form related to $\sigma^{a}$.

An explicit realisation of the asymptotic symmetry at null infinity was presented in [34] and turned out to be a semisimple enlargement of the $B M S_{3}$ algebra:

$$
\begin{aligned}
& {\left[\mathcal{J}_{m}, \mathcal{J}_{n}\right]=(m-n) \mathcal{J}_{m+n}+\frac{c_{1}}{12}\left(m^{3}-m\right) \delta_{m+n, 0},} \\
& {\left[\mathcal{J}_{m}, \mathcal{P}_{n}\right]=(m-n) \mathcal{P}_{m+n}+\frac{c_{2}}{12}\left(m^{3}-m\right) \delta_{m+n, 0},} \\
& {\left[\mathcal{P}_{m}, \mathcal{P}_{n}\right]=(m-n) \mathcal{Z}_{m+n}+\frac{c_{3}}{12}\left(m^{3}-m\right) \delta_{m+n, 0},} \\
& {\left[\mathcal{J}_{m}, \mathcal{Z}_{n}\right]=(m-n) \mathcal{Z}_{m+n}+\frac{c_{3}}{12}\left(m^{3}-m\right) \delta_{m+n, 0},} \\
& {\left[\mathcal{P}_{m}, \mathcal{Z}_{n}\right]=\frac{1}{\ell^{2}}(m-n) \mathcal{P}_{m+n}+\frac{c_{2}}{12 \ell^{2}}\left(m^{3}-m\right) \delta_{m+n, 0},} \\
& {\left[\mathcal{Z}_{m}, \mathcal{Z}_{n}\right]=\frac{1}{\ell^{2}}(m-n) \mathcal{Z}_{m+n}+\frac{c_{3}}{12 \ell^{2}}\left(m^{3}-m\right) \delta_{m+n, 0},}
\end{aligned}
$$

where the central charges $c_{1}, c_{2}$ and $c_{3}$ are related to the coupling constant of the CS action (B.2) as follows

$c_{1}=12 k \alpha_{0}, \quad c_{2}=12 k \alpha_{1}, \quad c_{3}=12 k \alpha_{2}$.

Such enlarged $B M S_{3}$ algebra results to be isomorphic to three copies of the Virasoro algebra. Indeed, by considering the following change of basis

$$
\begin{aligned}
& \mathcal{L}_{m}^{+}=\frac{1}{2}\left(\ell^{2} \mathcal{Z}_{m}+\ell \mathcal{P}_{m}\right), \mathcal{L}_{m}^{-}=\frac{1}{2}\left(\ell^{2} \mathcal{Z}_{-m}-\ell \mathcal{P}_{-m}\right), \\
& \hat{\mathcal{L}}_{m}=\mathcal{J}_{-m}-\ell^{2} \mathcal{Z}_{-m},
\end{aligned}
$$

one can rewrite the enlarged $B M S_{3}$ algebra as three copies of the Virasoro algebra,

$$
\begin{aligned}
& i\left\{\mathcal{L}_{m}^{+}, \mathcal{L}_{n}^{+}\right\}=(m-n) \mathcal{L}_{m+n}^{+}+\frac{c^{+}}{12} m^{3} \delta_{m+n, 0}, \\
& i\left\{\mathcal{L}_{m}^{-}, \mathcal{L}_{n}^{-}\right\}=(m-n) \mathcal{L}_{m+n}^{-}+\frac{c^{-}}{12} m^{3} \delta_{m+n, 0}, \\
& i\left\{\hat{\mathcal{L}}_{m}, \hat{\mathcal{L}}_{n}\right\}=(m-n) \hat{\mathcal{L}}_{m+n}+\frac{\hat{c}}{12} m^{3} \delta_{m+n, 0},
\end{aligned}
$$

with the following central charges

$c^{ \pm}=\frac{1}{2}\left(\ell^{2} c_{3} \pm \ell c_{2}\right), \quad \hat{c}=\left(c_{1}-\ell^{2} c_{3}\right)$.

It is interesting to note that the AdS-Lorentz algebra (B.1) is a finite subalgebra of the enlarged $B M S_{3}$ algebra (B.3). In fact, the finite set of generators $\left\{\mathcal{J}_{m}, \mathcal{P}_{m}, \mathcal{Z}_{m}\right\}$ with $m=0, \pm 1$ is related to the AdS-Lorentz ones through the following redefinitions

$$
\begin{aligned}
& \mathcal{J}_{-1}=-\sqrt{2} J_{0}, \mathcal{J}_{1}=\sqrt{2} J_{1}, \mathcal{J}_{0}=J_{2}, \\
& \mathcal{P}_{-1}=-\sqrt{2} P_{0}, \mathcal{P}_{1}=\sqrt{2} P_{1}, \mathcal{P}_{0}=P_{2}, \\
& \mathcal{Z}_{-1}=-\sqrt{2} Z_{0}, \mathcal{Z}_{1}=\sqrt{2} Z_{1}, \mathcal{Z}_{0}=J_{2} .
\end{aligned}
$$

\section{References}

1. J.D. Brown, M. Henneaux, Central charges in the canonical realization of asymptotic symmetries: an example from threedimensional gravity. Commun. Math. Phys. 104, 207 (1986)

2. A. Ashtekar, J. Bicak, B.G. Schmidt, Asymptotic structure of symmetry reduced general relativity. Phys. Rev. D 55, 669 (1997). arXiv:gr-qc/9608042

3. G. Barnich, G. Compere, Classical central extension for asymptotic symmetries at null infinity in three spacetime dimensions. Class. Quant. Grav. 24, F15 (2007). arXiv:gr-qc/0610130

4. G. Barnich, C. Troessaert, Aspects of the BMS/CFT correspondence. JHEP 1005, 062 (2010). arXiv:1001.1541 [hep-th]

5. H. Bondi, M.G.J. van der Burg, A.W.K. Metzner, Gravitational waves in general relativity. 7. Waves from axisymmetric isolated systems. Proc. Roy. Soc. Lond. A 269, 21 (1962)

6. R.K. Sachs, Gravitational waves in general relativity. 8. Waves in asymptotically flat space-times, Proc. Roy. Soc. Lond. A 270 , 103 (1962)

7. H.A. Gonzalez, J. Matulich, M. Pino, R. Troncoso, Asymptotically flat spacetimes in three-dimensional higher spin gravity. JHEP 1309, 016 (2013). arXiv:1307.5651 [hep-th]

8. H. Afshar, A. Bagchi, R. Fareghbal, D. Grumiller, J. Rosseel, Spin-3 gravity in three-dimensional flat space. Phys. Rev. Lett. 111(12), 121603 (2013). arXiv:1307.4768 [hep-th]

9. H.A. Gonzalez, M. Pino, Boundary dynamics of asymptotically flat 3D gravity coupled to higher spin fields. JHEP 05, 127 (2014). arXiv:1403.4898 [hep-th]

10. J. Matulich, A. Perez, D. Tempo, R. Troncoso, Higher spin extension of cosmological spacetimes in 3D: asymptotically flat behavior with chemical potentials and thermodynamics. JHEP $\mathbf{0 5}, 025$ (2015). arXiv:1412.1464 [hep-th]

11. O. Fuentealba, J. Matulich, R. Troncoso, Asymptotically flat structure of hypergravity in three spacetime dimensions. JHEP 10, 009 (2015). arXiv:1508.04663 [hep-th]

12. N. Banerjee, D.P. Jatkar, S. Mukhi, T. Neogi, Free-field realisations of the $\mathrm{BMS}_{3}$ algebra and its extensions. JHEP 06, 024 (2016). arXiv:1512.06240 [hep-th]

13. S. Detournay, M. Riegler, Enhanced Asymptotic Symmetry Algebra of 2+1 Dimensional Flat Space. Phys. Rev. D 95, 046008 (2017). arXiv:1612.00278 [hep-th]

14. M.R. Setare, H. Adami, Enhanced asymptotic $\mathrm{BMS}_{3}$ algebra of the flat spacetime solutions of generalized minimal massive gravity. Nucl. Phys. B 926, 70 (2018). arXiv:1703.00936 [hep-th]

15. A. Farmhand Parsa, H.R. Safari, M.M. Sheikh-Jabbari, On Rigidity of 3 d Asymptotic Symmetry Algebras, arXiv:1809.08209 [hepth] 
16. H.R. Safari, M.M. Sheikh-Jabbari, $\mathrm{BMS}_{4}$ algebra, its stability and deformations. JHEP 1904, 068 (2019). arXiv:1902.03260 [hepth]

17. P. Concha, N. Merino, O. Miskovic, E. Rodríguez, P. SalgadoRebolledo, O. Valdivia, Asymptotic symmetries of threedimensional Chern-Simons gravity for the Maxwell algebra. JHEP 10, 079 (2018). arXiv:1805.08834 [hep-th]

18. H. Bacry, P. Combe, J.L. Richard, Group-theoretical analysis of elementary particles in an external electromagnetic fields. 1. The relativistic particle in a constant and uniform field. Nuovo Cim. A 67, 267 (1970)

19. R. Schrader, The Maxwell group and the quantum theory of particles in classical homogeneous electromagnetic fields. Fortsch. Phys. 20, 701 (1972)

20. J. Gomis, A. Kleinschmidt, On free Lie algebras and particles in electro-magnetic fields. JHEP 07, 085 (2017). arXiv: 1705.05854 [hep-th]

21. J.A. de Azcarraga, K. Kamimura, J. Lukierski, Generalized cosmological term from Maxwell symmetries. Phys. Rev. D 83, 124036 (2011). arXiv:1012.4402 [hep-th]

22. R. Durka, J. Kowalski-Glikman, M. Szczachor, Gauges AdSMaxwell algebra and gravity. Mod. Phys. Lett. A 26, 2689 (2011). arXiv: 1107.4728 [hep-th]

23. J.A. de Azcarraga, K. Kamimura, J. Lukierski, Maxwell symmetries and some applications. Int. J. Mod. Phys. Conf. Ser. 23, 01160 (2013). arXiv:1201.2850 [hep-th]

24. P.K. Concha, D.M. Peñafiel, E.K. Rodríguez, P. Salgado, Evendimensional General Relativity from Born-Infeld gravity. Phys. Lett. B 725, 419 (2013). arXiv:1309.0062 [hep-th]

25. P.K. Concha, D.M. Peñafiel, E.K. Rodríguez, P. Salgado, ChernSimons and Born-Infeld gravity theories and Maxwell algebras type. Eur. Phys. J. C 74, 2741 (2014). arXiv:1402.0023 [hep-th]

26. P.K. Concha, D.M. Peñafiel, E.K. Rodríguez, P. Salgado, Generalized Poincaré algebras and Lovelock-Cartan gravity theory. Phys. Lett. B 742, 310 (2015). arXiv:1405.7078 [hep.th]

27. P. Salgado, R.J. Szabo, O. Valdivia, Topological gravity and transgression holography. Phys. Rev. D 89, 084077 (2014). arXiv: 1401.3653 [hep-th]

28. S. Hoseinzadeh, A. Rezaei-Aghdam, (2+1)-dimensional gravity from Maxwell and semisimple extension of the Poincaré gauge symmetric models. Phys. Rev. D 90, 084008 (2014). arXiv: 1402.0320 [hep-th]

29. O. Cebecioğlu, S. Kibaroğlu, Maxwell-affine gauge theory of gravity. Phys. Lett. B 751, 131 (2015). arXiv:1503.09003 [hep-th]

30. L. Avilés, E. Frodden, J. Gomis, D. Hidalgo, J. Zanelli, Nonrelativistic Maxwell Chern-Simons gravity. JHEP 1805, 047 (2018). arXiv:1802.08453 [hep-th]

31. J. Gomis, A. Kleinschmidt, J. Palmkvist, Symmetries of Mtheory and free Lie superalgebras. JHEP 03, 160 (2019). arXiv:1809.09171 [hep-th]

32. S. Kibaroğlu, M. Şenay, O. Cebecioğlu, $D=4$ topological gravity from gauging the Maxwell-special-affine group. Mod. Phys. Lett. A 34, 1950016 (2019). arXiv:1810.01635 [hep-th]

33. P. Salgado-Rebolledo, The Maxwell group in $2+1$ dimensions and its infinite-dimensional enhancements. arXiv:1905.09421 [hepth]

34. P. Concha, N. Merino, E. Rodríguez, P. Salgado-Rebolledo, O. Valdivia, Semi-simple enlargement of the $\mathfrak{b m s}_{3}$ algebra from a $\mathfrak{s o}(2,2) \oplus \mathfrak{s o}(2,1)$ Chern-Simons theory. JHEP 1902, 002 (2019). arXiv: 1810.12256 [hep-th]

35. D.V. Soroka, V.A. Soroka, Semi-simple extension of the (super)Poincaré algebra. Adv. High Energy Phys. 2009, (2009). arXiv:hep-th/0605251

36. J. Gomis, K. Kamimura, J. Lukierski, Deformations of Maxwell algebra and their dynamical realizations. JHEP 0908, 039 (2009). arXiv:0906.4464 [hep-th]
37. J. Díaz, O. Fierro, F. Izaurieta, N. Merino, E. Rodriguez, P. Salgado, O. Valdivia, A generalized action for $(2+1)$-dimensional Chern-Simons gravity. J. Phys. A: Math. Theor. 45, 255207 (2012). arXiv:1311.2215 [gr-qc]

38. P. Salgado, S. Salgado, so $(D-1,1) \otimes$ so $(D-1,2)$ algebras and gravity. Phys. Lett. B 728, 5 (2014)

39. P.K. Concha, R. Durka, C. Inostroza, N. Merino, E.K. Rodríguez, Pure Lovelock gravity and Chern-Simons theory. Phys. Rev. D 94, 024055 (2016). arXiv:1603.09424 [hep-th]

40. P.K. Concha, N. Merino, E.K. Rodríguez, Lovelock gravity from Born-Infeld gravity theory. Phys. Lett. B 765, 395 (2017). arXiv:1606.07083 [hep-th]

41. P. Concha, E. Rodríguez, Generalized pure Lovelock gravity. Phys. Lett. B 774, 616 (2017). arXiv:1708.08827 [hep-th]

42. P. Concha, E. Rodríguez, Non-relativistic gravity theory based on an enlargement of the extended Bargmann algebra. JHEP 07, 085 (2019). arXiv:1906.00086 [hep-th]

43. G. Barnich, L. Donnay, J. Matulich, R. Troncoso, Asymptotic symmetries and dynamics of three-dimensional flat supergravity. JHEP 1408, 071 (2014). arXiv:1407.4275 [hep-th]

44. I. Lodato, W. Merbis, Super-BMS 3 algebras from $\mathcal{N}=2$ flat supergravities. JHEP 1611, 150 (2016). arXiv:1610.07506 [hepth]

45. O. Fuentealba, J. Matulich, R. Troncoso, Asymptotic structure of $\mathcal{N}=2$ supergravity in $3 \mathrm{D}$ : extended super-BMS ${ }_{3}$ and nonlinear energy bounds. JHEP 1709, 030 (2017). arXiv:1706.07542 [hepth]

46. N. Banerjee, A. Bhattacharjee, Neetu, T. Neogi, New $\mathrm{N}=2$ SuperBMS $_{3}$ algebra and invariant dual theory for 3D supergravity. arXiv:1905.10239 [hep-th]

47. N. Banerjee, I. Lodato, T. Neogi, N=4 Supersymmetric $\mathrm{BMS}_{3}$ algebras from asymptotic symmetry analysis. Phys. Rev. D 96, 066029 (2017). arXiv:1706.02922 [hep-th]

48. N. Banerjee, A. Bhattacharjee, I. Lodato, T. Neogi, Maximmaly $\mathcal{N}$-extended super-BMS 3 algebras and Generalized 3D Gravity Solutions. JHEP 1901, 115 (2019). arXiv:1807.06768 [hep-th]

49. F. Izaurieta, E. Rodríguez, P. Salgado, Expanding Lie (super)algebras through Abelian semigroups. J. Math. Phys. 47, 123512 (2006). arXiv:hep-th/0606215

50. R. Caroca, P. Concha, E. Rodríguez, P. Salgado-Rebolledo, Generalizing the $b s_{3}$ and 2D-conformal algebra by expanding the Virasoro algebra. Eur. Phys. J. C 78, 262 (2018). arXiv:1707.07209 [hep-th]

51. R. Caroca, P. Concha, O. Fierro, E. Rodríguez, Three-dimensional Poincaré supergravity and $\mathcal{N}$-extended supersymmetric $\mathrm{BMS}_{3}$ algebra. Phys. Lett. B 792, 93 (2019). arXiv:1812.05065 [hepth]

52. S. Bonanos, J. Gomis, K. Kamimura, J. Lukierski, Maxwell superalgebra and superparticle in constant Gauge background. Phys. Rev. Lett. 104, 090401 (2010). arXiv:0911.5072 [hep-th]

53. P. Concha, D.M. Peñafiel, E. Rodríguez, On the Maxwell supergravity and flat limit in $2+1$ dimensions. Phys. Lett. B 785, 247 (2018). arXiv:1807.00194 [hep-th]

54. M. Hastsuda, M. Sakaguchi, Wess-Zumino term for the AdS superstring and generalized Inönü-Wigner contraction. Prog. Theor. Phys. 109, 853 (2003). arXiv:hep-th/0106114

55. J.A. de Azcarraga, J.M. Izquierdo, M. Picon, O. Varela, Generating Lie and gauge free differential (super)algebras by expanding Maurer-Cartan forms and Chern-Simons supergravity. Nucl. Phys. B 662, 185 (2003). arXiv:hep-th/0212347

56. J.A. de Azcarraga, J.M. Izquierdo, M. Picon, O. Varela, Extensions, expansions, Lie algebra cohomology and enlarged superspaces. Class. Quant. Grav. 21, S1375-1384 (2004). arXiv:hep-th/0401033 
57. J.A. de Azcarraga, J.M. Izquierdo, M. Picon, O. Varela, Expansions of algebras and superalgebras and some applications. Int. J. Theor. Phys. 46, 2734 (2007). arXiv:hep-th/0401033

58. R. Caroca, N. Merino, P. Salgado, S-expansion of higher-order Lie algebras. J. Math. Phys. 50, 013503 (2009). arXiv:1004.5213 [math-ph]

59. R. Caroca, N. Merino, A. Perez, P. Salgado, Generating higherorder Lie algebras by expanding Maurer Cartan forms. J. Math. Phys. 50, 123527 (2009). arXiv:1004.5503 [hep-th]

60. R. Caroca, N. Merino, P. Salgado, O. Valdivia, Generating infinitedimensional algebras from loop algebras by expanding MaurerCartan forms. J. Math. Phys. 52, 043519 (2011). arXiv:1311.2623 [math-ph]

61. R. Caroca, I. Kondrashuk, N. Merino, F. Nadal, Bianchi spaces and their three-dimensional isometries as S-expansions of two-dimensional isometries. J. Phys. A 46, 225201 (2013). arXiv:1104.3541 [math-ph]

62. L. Andrianopoli, N. Merino, F. Nadal, M. Trigiante, General properties of the expansion methods of Lie algebras. J. Phys. A 46, 365204 (2013). arXiv:1308.4832 [gr-qc]

63. M. Artebani, R. Caroca. M.C. Ipinza, D.M. Peñafiel, P. Salgado, Geometrical aspects of the Lie Algebra S-Expansion Procedure, J. Math. Phys. 57, 023516 (2016). arXiv:1602.04525 [math-ph]

64. M.C. Ipinza, F. Lingua, D.M. Peñafiel, L. Ravera, An analytic method for $S$-expansion involving resonance and reduction. Fortschr. Phys. 64, 854 (2016). arXiv: 1609.05042 [hep-th]

65. C. Inostroza, I. Kondrashuk, N. Merino, F. Nadal, On a Java library to perform S-expansions of Lie algebras. J. Phys: Conf. Ser. 1085, 052010 (2018). arXiv: 1802.04468 [math-ph]

66. C. Inostroza, I. Kondrashuk, N. Merino, F. Nadal, On the algorithm to find S-related Lie algebras. J. Phys: Conf. Ser. 1085, 052011 (2018). arXiv:1802.05765 [physics.comp-ph]

67. J. Lukierski, A. Nowicki, Superspinors and graded Lorentz groups in three, four and five dimensions. Fortsch. Phys. 30, 75 (1982)

68. N. Banerjee, D.P. Jatkar, I. Lodato, S. Mukhi, T. Neogi, Extended supersymmetric $\mathrm{BMS}_{3}$ algebras and their free field realisations. JHEP 11, 059 (2016). arXiv:1609.09210 [hep-th]

69. G. Barnich, L. Donnay, J. Matulich, R. Troncoso, Super-BMS 3 invariant boundary theory from three-dimensional flat supergravity. JHEP 1701, 029 (2017). arXiv:1510.08824 [hep-th]

70. A. Bagchi, I. Mandal, Supersymmetric extension of Galilean conformal algebras. Phys. Rev. D 80, 086011 (2009). arXiv:0905.0580 [hep-th]

71. I. Mandal, Supersymmetric extension of GCA in 2d. JHEP 1011, 018 (2010). arXiv:1003.0209 [hep-th]

72. C. Krishnan, A. Raju, S. Roy, A Grassmann path from $\mathrm{AdS}_{3}$ to flat space. J. High Energy Phys. 1403, 036 (2014). arXiv:1312.2941 [hep-th]

73. P. Concha, $\mathcal{N}$-extended Maxwell supergravities as Chern-Simons theories in three spacetime dimensions. Phys. Lett. B 792, 290 (2019). arXiv:1903.03081 [hep-th]

74. F. Izaurieta, E. Rodríguez, P. Minning, P. Salgado, A. Perez, Standard general relativity from Chern-Simons gravity. Phys. Lett. B 678, 213 (2009). arXiv:0905.2187 [hep-th]

75. S. Bonanos, J. Gomis, K. Kamimura, J. Lukierski, Deformations of Maxwell superalgebras and their applications. J. Math. Phys. 51, 102301 (2010). arXiv:1005.3714 [hep-th]

76. J. Lukierski, Generalized Wigner-Inönü contractions and Maxwell (Super)Algebras. Proc. Steklov Inst. Math. 272(1), 183 (2011). arXiv:1007.3405 [hep-th]

77. S. Fedoruk, J. Lukierski, New spinorial particle model in tensorial space-time and interacting higher spin fields. JHEP 1302, 128 (2013). arXiv:1210.1506 [hep-th]

78. J.A. de Azcarraga, J.M. Izquierdo, J. Lukierski, M. Woronowicz, Generalizations of Maxwell (super)algebras by the expansion method. Nucl. Phys. B 869, 303 (2013). arXiv:1210.1117 [hep-th]
79. J.A. de Azcarraga, J.M. Izquierdo, Minimal D $=4$ supergravity from superMaxwell algebra. Nucl. Phys. B 885, 34 (2014). arXiv: 1403.4128 [hep-th]

80. P.K. Concha, E.K. Rodríguez, Maxwell superalgebras and Abelian semigroup expansion. Nucl. Phys. B 886, 1128 (2014). arXiv: 1405.1334 [hep-th]

81. P.K. Concha, E.K. Rodríguez, $\mathrm{N}=1$ supergravity and Maxwell superalgebras. JHEP 1409, 090 (2014). arXiv:1407.4635 [hepth]

82. P.K. Concha, O. Fierro, E.K. Rodríguez, P. Salgado, ChernSimons supergravity in $\mathrm{D}=3$ and Maxwell superalgebra. Phys. Lett. B 750, 117 (2015). arXiv:1507.02335 [hep-th]

83. P.K. Concha, O. Fierro, E.K. Rodríguez, Inönü-Wigner contraction and $\mathrm{D}=2+1$ supergravity. Eur. Phys. J. C 77, 48 (2017). arXiv:1611.05018 [hep-th]

84. D.M. Peñafiel, L. Ravera, On the hidden Maxwell Superalgebra underlying D = 4 supergravity. Fortsch. Phys. 65, 1700005 (2017). arXiv:1701.04234 [hep-th]

85. L. Ravera, Hidden role of Maxwell superalgebras in the free differential algebras of $D=4$ and $D=11$ supergravity. Eur. Phys. J. C 78, 211 (2018). arXiv:1801.08860 [hep-th]

86. S. Kibaroğlu, O. Cebecioğlu, $D=4$ supergravity from the Maxwell-Weyl superalgebra. arXiv:1812.09861 [hep-th]

87. M.B. Green, Supertranslations, superstrings and Chern-Simons forms. Phys. Lett. B 223, 157 (1989)

88. R. D'Auria, P. Fré, Geometric supergravity in $\mathrm{d}=11$ and its hidden supergroup. Nucl. Phys. B 201, 101 (1982)

89. R. Basu, S. Detournay, M. Riegler, Spectral flow in 3D flat spacetimes. JHEP 12, 134 (2017). arXiv:1706.07438 [hep-th]

90. K. Ito, Extended superconformal algebras on AdS(3). Phys. Lett. B 449, 48 (1999). arXiv:hep-th/9811002

91. P.K. Concha, E.K. Rodríguez, P. Salgado, Generalized supersymmetric cosmological term in N=1 Supergravity. JHEP 08, 009 (2015). arXiv:1504.01898 [hep-th]

92. P.K. Concha, M.C. Ipinza, L. Ravera, E.K. Rodríguez, On the supersymmetric extension of Gauss-Bonnet like gravity. JHEP 09, 007 (2016). arXiv:1607.00373 [hep-th]

93. A. Baunadi, L. Ravera, Generalized AdS-Lorentz deformed supergravity on a manifold with boundary. Eur. Phys. J. Plus 133, 514 (2018). arXiv: 1803.08738 [hep-th]

94. D.M. Peñafiel, L. Ravera, Generalized cosmological term in $D=$ 4 supergravity from a new AdS-Lorentz superalgebra. Eur. Phys. J. C 78, 945 (2018). arXiv:1807.07673 [hep-th]

95. O. Fierro, F. Izaurieta, P. Salgado, O. Valdivia, Minimal AdSLorentz supergravity in three-dimensions. Phys. Lett. B 788, 198 (2019). arXiv:1401.3697 [hep-th]

96. D.V. Soroka, V.A. Soroka, Tensor extension of the Poincaré algebra. Phys. Lett. B 607, 302 (2005). arXiv:hep-th/0410012

97. I. Manda, A. Rayyan, Super-GCA from $\mathcal{N}=(2,2)$ SuperVirasoro. Phys. Lett. B 754, 195 (2016). arXiv:1601.04723 [hepth]

98. E. Bergshoeff, J. Izquierdo, T. Ortín, L. Romano, Lie algebra expansions and actions for non-relativistic gravity. JHEP 08, 048 (2019). arXiv:1904.08304 [hep-th]

99. J.A. de Azcárraga, D. Gútiez, J.M. Izquierdo, Extended $D=3$ Bargmann supergravity from a Lie algebra expansion. Nucl. Phys. B 946, 114706 (2019). arXiv:1904.12786 [hep-th]

100. L. Romano, Non-relativistic four dimensional p-Brane supersymmetric theories and Lie algebra expansion. arXiv:1906.08220 [hep-th]

101. D.M. Peñafiel, P. Salgado-Rebolledo, Non-relativistic symmetries in three space-time dimensions and the Nappi-Witten algebra. Phys. Lett. B 798, 135005 (2019). arXiv:1906.02161 [hep-th]

102. S. Bansal, D. Sorokin, Can Chern-Simons or Rarita-Schwinger be a Volkov-Akulov Goldstone? JHEP 07, 106 (2018). arXiv:1806.05945 [hep-th] 
103. D. Chernyavsky, D. Sorokin, Three-dimensional (higher-spin) gravities with extended Schrödinger and 1-conformal Galilean symmetries. JHEP 07, 156 (2019). arXiv:1905.13154 [hep-th]

104. J. Hietarinta, Supersymmetry generators of arbitrary spin. Phys. Rev. D 13, 838 (1976)

105. P. Concha, L. Ravera, E. Rodríguez, On the supersymmetry invariance of flat supergravity with boundary. JHEP 01, 192 (2019). arXiv:1809.07871 [hep-th]
106. R. Caroca, P. Concha, O. Fierro, E. Rodríguez, P. SalgadoRebolledo, Generalized Chern-Simons higher-spin gravity theories in three dimensions. Nucl. Phys. B 934, 240 (2018). arXiv:1712.09975 [hep-th]

107. R. Durka, J. Kowalski-Glikman, Resonant algebras in ChernSimons model of topological insulators. Phys. Lett. B 795, 516 (2019). arXiv:1906.02356 [hep-th] 